\title{
Kernos
}

Revue internationale et pluridisciplinaire de religion grecque antique

15 | 2002

Varia

\section{La théogonie orphique du papyrus de Derveni}

\section{Alberto Bernabé}

Édition électronique
URL : http://journals.openedition.org/kernos/1370

DOl : 10.4000/kernos. 1370

ISSN : 2034-7871

\section{Éditeur}

Centre international d'étude de la religion grecque antique

Édition imprimée

Date de publication : 1 janvier 2002

ISSN : 0776-3824

\section{Référence électronique}

Alberto Bernabé, «La théogonie orphique du papyrus de Derveni », Kernos [En ligne], 15 | 2002, mis en ligne le 21 avril 2011, consulté le 02 mai 2019. URL : http://journals.openedition.org/kernos/1370 ; DOI : 10.4000/kernos. 1370 


\section{La théogonie orphique du papyrus de Derveni*}

\section{Objectif}

Nous avons l'intention dans cet article d'offrir une synthèse de nos connaissances actuelles sur la théogonie attribuée à Orphée et commentée dans le Papyrus de Derveni. On dispose d'une longue série de travaux sur cet important document littéraire - dus à la plume de spécialistes d'un très grand renom - notamment ceux qui ont été présentés dans un colloque tenu à Princeton et récemment publiés ${ }^{1}$, et de quelques nouvelles données sur le texte que l'on connaissait ${ }^{2}$. Nous sommes dès lors en état, à ce qu'il me semble, d'aborder une révision des problèmes concernant l'interprétation de cet ouvrage, d'en souligner les aspects qui jouissent aujourd'hui d'un large consensus et d'y relever les problèmes interprétatifs toujours à résoudre, tout en proposant quelques solutions ${ }^{3}$.

\section{Le papyrus de Derveni}

\subsection{Une importante découverte ${ }^{4}$}

En 1962 on découvre à Derveni, à quelque dix $\mathrm{km}$ au nord-ouest de Salonique, auprès d'un tombeau faisant partie d'un groupe de six, parmi les restes d'un bûcher funéraire, un rouleau de papyrus à demi calciné, destiné sans doute à être brûlé au bûcher du mort avec d'autres objets que celui-ci devait affectionner particulièrement ${ }^{5}$. Miraculeusement, jusqu'à vingt-six

1 Laks - Most (1997).

2 Généreusement fournies par le Professeur K. Tsantsanoglou, $c f . \S 2.1 . C f$. aussi les contributions à l'étude du texte apportées par Betegh (1999).

3 Pour des renseignements bibliographiques sur le papyrus, cf. Bernabé (1992a), 3335, et, pour plus de détails, $c f$. Funghi (1995) et (1997b). $C f$. aussi Funghi (1997a) pour une information générale exacte à son sujet et Bernabé (2001b) pour une traduction en espagnol annotée.

4 Sur des questions archéologiques se rattachant au papyrus, cf. Hood (1961-1962), Blake (1961-1962), Vanderpool (1962) 390 et fig.107-108, Daux (1962), Oschenschlager (1963), Hunger (1962), Makaronas (1963), Bingen (1967), Bottini (1992) 135-148.

5 Il paraît même que le mort devait lui attribuer un certain pouvoir talismanique tout comme une espèce de passeport pour l'au-delà - pareil d'ailleurs à celui que les lamelles en or semblent posséder, $c f$. Casadio (1994) 215, Scalera McClintock (1988) 141. On peut se rappeler à ce sujet l'exemple du papyrus trouvé dans les mains d'un défunt dans un tombeau à Callatis (Kollet) et malheureusement détruit dès qu'il en fut retiré, de telle sorte qu'il ne put jamais être lu, $c f$. Pippidi (1967) 209, Condurachi (1975) $184 s q$, 
colonnes du texté contenu dans ce papyrus nous sont parvenues, bien qu'incomplètes, car la partie inférieure commune à toutes a été entamée par le feu. On peut donc y lire passablement bien les dix ou douze premières lignes (parfois même quinze) de chaque colonne, viennent ensuite quelques lignes en très mauvais état qui devaient être suivies, à leur tour, de celles qui sont perdues, dont le chiffre exact ne peut être précisé. Nous ignorons également si le texte se poursuivait sur un deuxième rouleau lequel, avec moins de fortune, aurait complètement brûlé $(c f . \$ 6.14)$. Plusieurs raisons paléographiques et archéologiques ont conduit les experts à fixer la date du rouleau entre 340 et 320 av. J.-C. ${ }^{6}$, une date exceptionnellement haute pour les papyrus écrits en grec qui nous sont parvenus.

Une véritable édition complète du Papyrus, à laquelle travaille le philologue grec $\mathrm{K}$. Tsantsanoglou, n'a pas encore été publiée. Nous ne possédons qu'une copie du texte, dépourvue d'appareil critique, publiée dans $Z P E$ $47,1982,{ }^{*} 1 s q$. (après la p. 300) qui se fonde sur une première version, enrichie par quelques suppléments de Tsantsanoglou et de Parassoglou, mais présentant des additions de provenance très diverse, avec des lectures déjà périmées et un grand nombre d'erreurs, ce qui a conduit à la désavouer? Nous y ferons allusion dorénavant en employant l'abréviation ZPE (1982). Betegh présente, à son tour, un texte du même Papyrus qui ne cherche pas à être une édition critique. Il a tout simplement essayé de « réunir les informations les plus actualisées dont on puisse disposer » à son sujet, complétées par quelques lectures de son $\mathrm{cru}^{8}$. D'un autre côté, nous disposons encore de quelques éditions de différents passages du texte?. A l'occasion de mon travail sur l'édition critique de différents fragments orphiques, qui doit être

Schmidt (1975) 230, West (1983) 25, et aussi celui d'une amphore apulienne, aux figures en rouge, de 330-320 av. J.-C., du Peintre de Ganymède (Bâle Antikenmuseum Cat. 214), où Orphée se trouve devant un défunt assis qui tient un papyrus dans sa main gauche. $C f$. aussi R. Olmos et Bernabé-Jiménez San Cristóbal (2002) 292-295.

6 C'est la datation de Tsantsanoglou et Parássoglou (1988) 125 et (1992) 221; en outre ce papyrus a été daté par Turner (1971) 92, entre 325 et 275 av. J.-C., et d'après Youtie, il daterait du milieu du Iv $^{\mathrm{e}}$ siècle av. J.-C., tel qu'il l'indique dans une note ajoutée à Blake (1961-1962) par Robinson. Cet avis est partagé par Makaronas et Vanderpool (1962) 390, et Turner (1980) 26, cf. encore les propositions plus anciennes et moins précises de Kapsomenos (1964-1965) (avec les discussions d'un groupe d'experts) et de Speyer (1970) 44,46 n. 14.

7 Cf. Turner, Tsantsanoglou et Parássoglou (1982). Le texte de $Z P E$ est reproduit dans Mandilaras (1994) 32 sq.; 429-439.

8 Betegh (1999).

9 Kapsomenos (1963) (une partie des colonnes XVII et XXII et quelques mots isolés), (1964) (colonnes XIV-XX), Tsantsanoglou et Parássoglou (1988) (col. I), (1992), mais surtout les sept premières colonnes, avec commentaire, éditées par Tsantsanoglou (1997). Il faut encore signaler que la numérotation des colonnes a changé. En effet, les références antérieures à cette dernière publication commençaient leur numérotation à la colonne qui est aujourd'hui marquée du numéro $V$, il a donc fallu ajouter quatre numeros aux numérotations antérieures afin qu'elles correspondent au dernier état. 
publiée par la Bibliotbeca Teubneriana, j'ai naturellement décidé d'y inclure ces fragments, mais je ne pouvais suivre le texte désavoué. Le professeur Tsantsanoglou a eu la gentillesse - dont je lui suis très reconnaissant - de réviser la première version de mon édition des passages choisis et de m'offrir à leur sujet de nombreuses nouvelles lectures et d'autres suggestions. C'est donc grâce à tout ceci que j'ai pu disposer d'un texte fiable. La soigneuse traduction en anglais d'un texte, également révisé par Tsantsanoglou, publiée par Laks et Most dans le volume collectif auquel je me suis déjà référé ${ }^{10}$ et aussi l'excellente traduction de Janko (2001), pourvue d'un apparatus avec de nouvelles lectures et interprétations ont été également pour moi d'une grande utilité. Les auteurs m'ont permis de lire leurs traductions avant qu'elles ne soient publiées, ce qui m'a donné la possibilité d'avancer considérablement mon travail. De son côté Betegh m'a fait parvenir un exemplaire de sa thèse de doctorat (Betegh 1999). Ma gratitude à leur égard est également très grande.

\subsection{Un texte complexe}

La seule conservation du papyrus, due aux curieuses circonstances auxquelles je me suis déjà référé, fait de celui-ci un document unique, mais il l'est encore davantage par le type de texte qu'il contient. Son auteur y commente un passage d'Héraclite, puis analyse quelques rituels et disserte sur les Érynies avant d'aborder, en continu, un long commentaire de quelques vers qu'il attribue à Orphée. À un moment donné, il cite dans son commentaire un vers comme appartenant aux Hymnes du même auteur, mais la plupart de ceux dont il fait mention feraient partie d'un poème sur l'organisation du monde et la lutte qui s'engage entre plusieurs dieux pour détenir le suprême pouvoir. C'est ce qu'on a appelé, aussi improprement qu'on voudra, Théogonie. Ce qui est encore plus curieux, c'est que l'auteur de l'œuvre en prose y cite aussi trois vers qui ressemblent beaucoup à des vers que nous connaissions déjà dans l'œuvre d'Homère ${ }^{11}$.

\footnotetext{
10 Laks - Most (1997).
}

11 Concrètement un vers parlant d'Hermès, très similaire à Odyssée VIII, 335, et deux autres qui abordent le thème de la jarre des biens et des maux, coïncidant, plus dans leur contenu que dans leur forme, avec Iliade XXIV, 527 sq. La plupart des auteurs ont considéré qu'il s'agissait tout simplement, dans ces deux derniers cas, de deux citations d'Homère ayant subi les altérations propres à la transmission ancienne. Or le commentateur se réfère à ces vers dans son texte en les attribuant clairement à Orphée, car il les introduit au moyen des expressions "et montre »... " et montre », sans indication explicite de changement de sujet, de telle manière que ce rôle syntaxique doit bien continuer à être rempli par Orphée, l'auteur également, d'après le commentateur, de tous les autres vers qu'il cite dans son commentaire. D'après Funghi (1995) 574, il s'agirait de vers qui appartiendraient à un fonds épique traditionnel, pouvant donc avoir été employés par plus d'un poète. Pour Obbink (1997) $41 \mathrm{n}$. 4, le commentateur semble considérer Orphée comme l'auteur des vers en question; ce serait alors Homère qui les lui aurait empruntés. Cf. également la mise au point mesurée présentée par Betegh (1999) 
Mais, laissant de côté ces vers « homériques » et la citation d'Héraclite, dont nous n'allons pas nous occuper à présent, il reste encore à considérer dans ce document deux textes de différente nature qui appartiennent aussi à deux époques différentes; le plus récent des deux est le commentaire en prose, qui, comme on verra, doit remonter à une époque très proche de 400 av. J.-C. et le plus ancien, probablement antérieur à 500 av. J.-C., qui est constitué par les vers au sujet desquels le commentateur construit son commentaire, en les attribuant à Orphée.

\section{Le poème d'Orphée cité dans le commentaire}

Nous ne possédons du poème orphique qu'une série de citations, plus ou moins longues. West ${ }^{12}$ fit une reconstruction exempli gratia du poème, dans laquelle il transformait les trente vers ou demi vers originaux sans connexion en un ouvrage composé de 47 vers suivis. Cette reconstitution est ingénieuse et brillante, et même très vraisemblable par endroits, mais on ne doit lui accorder d'autre statut que celui que l'auteur lui-même lui confère, celui de la pure possibilité, frôlant presque le simple amusement philologique ${ }^{13}$.

On a appelé ce poème Théogonie ${ }^{14}$ par convention, parce qu'il fait référence à des récits mythiques qui remontent à l'origine des temps et que nous retrouvons en partie dans d'autres théogonies, dont celle d'Hésiode et des orphiques ultérieurs. Or, dans les vers qui en ont été conservés, nous voyons comment le poète passe rapidement sur des parties importantes du sujet, en s'y référant sommairement au moyen de relatifs. Cette "vitesse narrative " constitue une formule typique déjà employée dans des textes courts, qui n'ont de sens que s'ils en présupposent d'autres appartenant au même univers mythique. Ce recours constant à l'intertextualité est caractéristique de la littérature religieuse grecque (et de celle de toute époque). Bien que ce soit en nous mouvant sur le terrain glissant des hypothèses seulement vraisemblables, nous pourrions souligner plusieurs traits du poème : il n'aurait pas de prétention à la systématisation, comme la théogonie d'Hésiode, et

$\$ 1.2$ et Janko (2001) 31 n. 186 et 32 n. 187 ( « if these Homeric verses were reused in a Orphic poem, just as the Homeric Hymn to Demeter was reused in the Orphic poem in Berlin papyrus 13044 "). En tout cas, tout ceci ne devrait pas mener aux conclusions extrêmes et inadmissibles de Böhme (1988) et (1989).

12 West (1983) $114 s q$.

13 Le problème est que quelques auteurs ont travaillé avec cette reconstitution comme s'il s'agissait d'un poème authentique, par exemple Scalera Mcclintock (1988), Brisson (1993) 57 sq. Le cas le plus curieux que j'en connaisse est celui de Sorel (1995) 58 $s q$. qui cite comme «P. Derv. v. 31-32» les vers de la reconstitution de West. $C f$. aussi la notice de Y. Vernière dans Mentor, Liège 1992 n. 2004 p. 724 : « l'A. donne pour la première fois une transcription complète du Papyrus de Derveni » (1). Je souscris à l'observation de Calame (1991) 229 n. 4, dans le sens que le charme de cette reconstitution ne devrait pas instaurer l'habitude de ne pas retourner directement aux textes.

14 Alderink (1981) 26 l'appelle « Theological cosmology ». Janko (1986) 158, de son

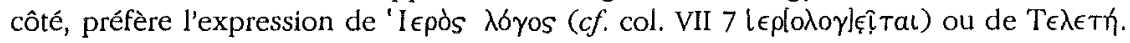


ce ne serait pas un texte long ${ }^{15}$, car il ne se serait centré que sur quelques aspects d'un mythe théogonique, à savoir la prise du pouvoir divin par Zeus et sa recréation du monde, alors que les antécédents de cette recréation, les premiers dieux et leur lutte pour le pouvoir, n'y seraient que des allusions. Un tel point de départ implique déjà que l'auteur présuppose l'existence d'autres récits mythiques (orphiques ou non : la Théogonie d'Hésiode pouvant en être un), il semble donc que ce soit plus pertinent à cet égard de considérer ce texte comme un court poème qui en présuppose d'autres que comme le poème abrégé d'un poème antérieur ${ }^{16}$. Les ouvrages les plus proches de celui-ci qui puissent être indiqués ici seraient, d'un côté, les Hymnes bomériques anciens et, de l'autre, ce qu'on a appelé le Testament d'Orpbée,

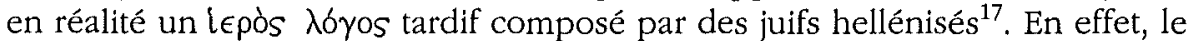
commentateur l'appelle « hymne ${ }^{18}$ tandis que Janko ${ }^{19}$ préfère le considérer

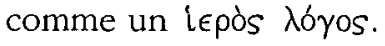

Le texte présente des coïncidences hautement significatives avec d'autres poèmes orphiques tardifs, ce qui nous indique qu'il a existé une tradition orphique plus ancienne que l'hypercritique des années cinquante à soixante ne l'a prétendu. Mais nous ne devons pas pour autant nous tenir à l'extrême opposé, c'est-à-dire celui de minimiser les différences, non moins significatives, que notre version présente à l'égard d'autres ouvrages postérieurs et dont la longueur ne serait pas la moins importante, car, par exemple, ce qu'on a appelé les Rhapsodies étaient composées de 24 chants, c'est-à-dire une longueur similaire à celle de l'Iliade ou l'Odyssée.

Si l'on envisage l'ordre dans lequel notre commentateur présente les citations, le poème s'ouvrirait par un chant à la gloire de Zeus pour remonter ensuite - de façon très sommaire et allusive, certes, comme je l'ai déjà souligné - à des époques antérieures au règne de Zeus et s'achever sur la curieuse recréation du monde menée à bien par le dieu. Cette manière de raconter, en Ringkomposition, n'est pas celle que nous connaissions dans les Théogonies, lesquelles adoptent généralement un ordre d'exposition linéaire, tout en se conformant à l'ordre de succession des événements ${ }^{20}$. Il existe cependant des divergences considérables parmi les spécialistes qui reconstituent la suite

15 Tsantsanoglou (1997) 118 n. 46 considère qu'il serait composé de 80 vers environ.

16 Tout comme fait West (1983) 87,95 n. 44, qui le considère comme l'abrégé d'une Théogonie de Protogonos antérieure.

17 Cf. Riedweg (1993).

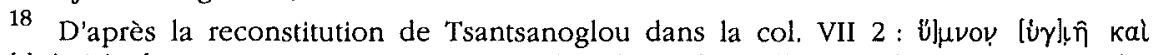
$\theta \epsilon \mu(\iota)$ Tà $\lambda \epsilon ́ \gamma o[\nu T a$, c'est à sa suite que Most (1997) 125 l'appelle «Orphic hymn ». Sur les difficultés de le considérer ainsi $c f$. Betegh (1999) $₫ 2.1$.

19 Janko (1986) 158.

20 Comme l'observe Betegh (1999) $\$ 2.1$. 
événementielle du poème concernant l'interprétation de quelques aspects de son contenu ${ }^{21}$.

\section{Le commentaire et son auteur anonyme}

Le commentaire est très particulier. Même si son auteur y emploie une terminologie exégétique extrêmement fine, qui peut surprendre dans un document aussi ancien, il ne l'aborde pas pour autant d'un point de vue philologique ou littéraire. Il y essaie, par contre, d'expliquer le sens véritable de ces vers, convaincu que le poète, Orphée, tout en ayant adopté un langage commun, y emploie les mots dans un sens allégorique, pour qu'il ne soient compris que de quelques-uns ${ }^{22}$. Aussi prend-il position dans un courant allégoriste d'interprétation des poètes dont nous avions déjà quelque indice mais dont l'évidence d'un texte représentatif aussi ancien nous manquait. C'est ainsi que notre auteur se serait servi dans son commentaire d'arguments philosophiques, ou pseudo-philosophiques, pour « expliquer » le « sens véritable » que contiennent, d'après lui, les vers qu'il cite.

Mais le fait que l'auteur, en plus de commenter ces vers, fasse référence dans son texte à d'autres sujets a soulevé une certaine controverse lors de la

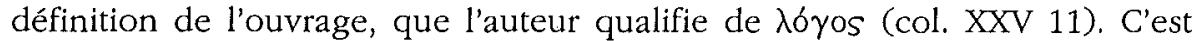
ainsi qu'on en a parlé comme d'un commentaire ${ }^{23}$, d'une exégèse allégorique $^{24}$ ou d'un traité Sur les rituels ${ }^{25}$, alors que d'autres spécialistes se sont montrés beaucoup plus prudents à l'heure de le qualifier ${ }^{26}$. Quoi qu'il en soit, et bien que ce type de commentaire ne nous aide pas trop, habituellement, à interpréter le poème ancien qu'il cite, il n'en reste pas moins qu'il possède un énorme intérêt en lui-même, car il présente des influences bien claires d'Anaxagore, Héraclite, Leucippe et Diogène d'Apollonie ${ }^{27}$. Par contre, notre auteur semble ignorer complètement Platon. Ces traits ont amené les experts

21 Cf. Funghi (1979), Alderink (1981) 36 sq., Kirk - Raven - Schofield (1983), West (1983) 75 sq., Brisson (1985), Burkert (1987) 22, (1998) 389 sq., Scalera McClintock (1988) 139 sq., Bernabé (1989), Eggers Lan (1991) 103 sq., Edwards (1991), Casadesús (1995) 192 sq., (1996), Baumgarten (1998) $98 s q$.

22 Cf. $\$ 5$, et Bernabé (1999a).

23 Kapsomenos (1963), Pfeiffer (1968) 139 n. 1, Turner (1980) 205, West (1983) 78; 80, Henry (1986) 150. C'est un commentaire philosophique pour Burkert (1970) 443, et systématique pour Lamedica (1991) 83. Cet avis n'est pas partagé par Tsantsanoglou, comme il l'explique dans sa lettre à Funghi, cf. Funghi (1995) 573.

24 Vian (1984).

25 Burkert (1986) 5, Tsantsanoglou per litteras à Funghi (1995) 573.

26 «Monografia pre-alessandrina su un testo orfico », selon Wilson (1983) 85; « discussion of Orphic Texts » selon Wilson (1984) 105.

27 Burkert (1968) 93 sq., (1970) 443 sq., West (1983) 80 sq., Laks (1997) 121 sq., Janko (1997) $63 s q$. 
à dater le texte de l'époque à laquelle je me suis référé plus haut, vers 400 av. J.-C. environ ${ }^{28}$.

Nous pouvons, ainsi donc, considérer le commentateur, par sa formation et la manière dont il s'exprime, comme l'un des derniers présocratiques, quoique ce soit très difficile, voire impossible, d'établir son identitée ${ }^{29}$. Ce qui reste bien clair, en tout cas, c'est qu'il représente une manière de travailler qui était toujours en vigueur à l'époque de Platon, tel qu'on peut l'apprécier dans plusieurs de ses dialogues, notamment le Cratyle et l'Euthyphron ${ }^{30}$. On a également prouvé que ce n'était pas un texte réservé aux cercles orphiques, ni secret, mais qu'il aurait joui par contre d'une certaine fortune, car il fut connu aussi d'autres auteurs et même cité par ceux-ci, dont Philochore et un scholiaste d'Hésiode ${ }^{31}$.

La discussion s'est encore portée sur la question de savoir si notre auteur était orphique ${ }^{32}$, dans la mesure où il cite et explique un poème d'Orphée, ou anti-orphique ${ }^{33}$, car son explication en prend un biais physique et non théologique. En réalité cette discussion ne devrait pas se poser en des termes aussi tranchants. Il y a beaucoup de manières d'être « orphique » et notre commentateur, du moment où il recherche le «sens véritable » du texte d'Orphée, peut être considéré comme orphique, même si sa formation et ses préférences l'amènent à effectuer un commentaire du texte de caractère

29 On a parlé à ce propos d’Épigénès (Kapsomenos [1964-5] 12; cette hypothèse est suivie par Edwards [1991] 205, mais contestée par Janko [1997] 71); de Métrodôros (Burkert cité par Ricciardelli Apicella [1980] 129: de son côté, Pfeiffer [1968] 237, West [1983] 82 et Long [1992] 65 n. 53 se réfèrent aussi à cet auteur lorsqu'ils parlent du papyrus, mais Sider [1997] 138, Janko [1997] 76 sq. réfutent cette idée); ou de Stésimbrote (Burkert [1986], $c f$. Tsantsanoglou per litteras dans Funghi [1995] 584 et la critique de Janko [1997] $73 s q$ ), dont nous ne connaissons que le nom et quelque référence isolée et, encore, d'autres auteurs mieux connus, dont Euthyphron, un personnage des Dialogues de Platon (cf. Kahn [1973] 156 n. 6, [1986] 98 sq., [1997] 55 sq., et Boyancé [1974] 109 sq., Tsantsanoglou per litteras in Punghi [1995] 584, mais Baxter [1992] 132 sq. et Janko [1997] 72 critiquent cette proposition), ou le sophiste Prodikos de Keos ( $c f$. Lebedev dans Sider [1997] 129 n. 2 et dans Janko [1997] 75). Janko (1997) fait aussi mention d'Anaximandre (non le philosophe mais l'historien, cf. FGrHist 9) et de Glaukôn, aux p. 75 sq.; de Diagoras de Mélos aux p. $87 \mathrm{sq}$. ( $c f$. aussi Janko [2001]) et de Diogène d'Apollonie aux p. 80 sq. $C f$., en plus de la bibliographie déjà citée, Boyancé (1974) 108 sq., West (1983) 78 sq., Casadesús (1995) $170 s q$. , Calame (1997) 75, Sider (1997) 129 n. 2.

30 Zhmud' (1983) 138 sq., Funghi (1995) 584, Richardson (1975) 65 sq.

31 Cf. Philochore (in Philodem., De piet., P. Hercul. 1428, fr. 3, 13 sq. +248 II 1 sq., p. 63 et 23 Gomperz), comme Obbink (1994) l'a prouvé, cf. aussi Obbink (1997) 49 n. 16, et Schol. Hes. Op. 63b (37, 3 Pertusi) (= Metrodor., 61, 6 D.-K., cf. Orph., fr. 347 Kern), au sujet duquel $c f$. Burkert (1970) 443 n. 1; voir aussi Funghi (1995) 573.

32 Alderink (1981) 27 et West (1983) $78 \mathrm{sq}$. le soutenaient.

33 C'est ce que Henrichs (1984) 255 ou Casadio (1987) 386 prétendent. Reale le considère comme un Pythagoricien éclectique, dans sa note à Arist. De mund. 274 (la deuxième édition de cet ouvrage, de Reale et Bos, p. 349). 
philosophique et étymologique et à en biaiser extrêmement l'interprétation. L'un des apports fondamentaux du récent colloque de Princeton est justement d'avoir montré comment l'allégorie physique se trouve dans ce texte au service d'un discours essentiellement religieux et initiatique ${ }^{34}$.

L'auteur nous y est donc présenté comme l'un de ces personnages auxquels Platon ${ }^{35}$ se réfère dans son texte :

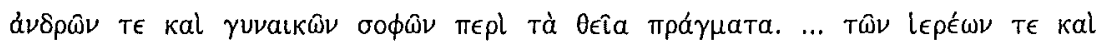

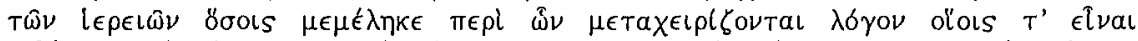

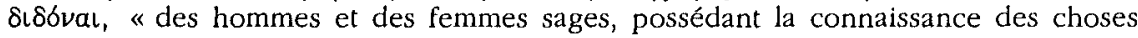
divines, des prêtres et des prêtresses qui jugent important de donner une explication sur ce dont ils s'occupent et qui sont capables de le faire ».

\section{La méthode d'analyse du commentateur ${ }^{36}$}

Dans le but de déterminer le degré d'utilité que peuvent avoir pour nous les apostilles ajoutées par le commentateur au texte, il convient que nous nous arrêtions un moment sur sa méthode d'analyse du texte. Commençons par lire l'un de ses passages, particulièrement révélateur ${ }^{37}$ à cet égard :

34 Cf. Zhmud" (1983) 139 (" adapting himself to the demands of his time, the author ... tries to gloss over the unsightly moral impression left upon the reader by the poem itself »), Laks et Most (1997) 4 (" the physical allegoresis ... was in the service of a project which was religious, and more particularly initiatory, in character »), Obbink (1997) 52 (" he ... acknowledges Orpheus as a spiritual authority, to whose books he imparts a ... message fathomable only by a learned élite »); Calame (1997) 76 (« these new readings reinforce the semantic coherence which inscribes the exegesis of the poem within the sequence of the description of the rites »; $c f$. 79), West (1997) 84 ( « he [sc. commentator] is their learned exegete ... who expounds to them [sc. the initiates of an Orphic-Bacchic cult societyl the meaning of what they do and say "; $c f$. déjà (1983) 81 " it is not unreasonable to conjecture ... he was himself one of the initiates whose ritual acts he knows and interprets 》); Tsantsanoglou (1997) 99 (« his purpose was to divulgate his professional secrets to the faithful »); $c f$. aussi Funghi (1995) 585, Casadesús (1995) 493, Bernabe (1997b) 37 sq., Most (1997) 120 « he is ... the leader of a particular grouping or sect within Orphism which consider itself Orphic and stand in opposition to nonOrphics, but at the same time distinguishes itself by its doctrine from other Orphic groups » et 122 " he is not a Presocratic, buth rather an Orphic who cannot ignore Presocratic thought », Janko (2001) 12 «effort to reconcile science and religion by means of allegory and etymology ».

35 Ménon, 81b, cf. Bernabé (1999b). Les rapports de l'auteur du commentaire avec le type de personnages cités par Platon ont été soulignés par West (1997) 84.

36 Cf. Burkert (1970) 444 sq., Richardson (1975), Prontera (1978) 48 sq., Schwabl (1978) 1327 sq., Ricciardelli Apicella (1980), Gambarara (1984a) 109 sq., (1984b) 168; 176ss; 180; 203 sq., Henry (1986), Lamedica (1991) 87 sq., Bernabé (1992b) 51 sq., (1995) 225 sq. (1996a) 63 sq., (1997a) 55 sq., Casadesús (1995) 235ss; 478 sq. Obbink (1997) 42, Tsantsanoglou (1997) 122, Most (1997) 123 sq., Laks (1997) 134 sq. Pour la comparaison de ses méthodes d'analyse avec celles de Platon dans le Cratyle, $c f$. Burkert (1970), Baxter (1992), Lamedica (1989), (1991) Most (1997), Laks (1997), Casadesús (2000) 56-60.

37 Cf. l'analyse approfondie du passage effectuée par Tsantsanoglou (1997) 117 sq. 


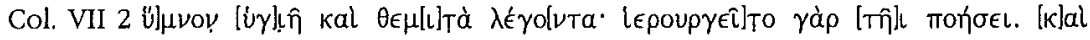

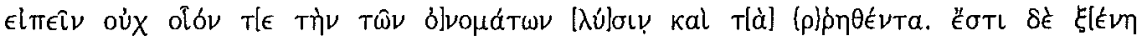

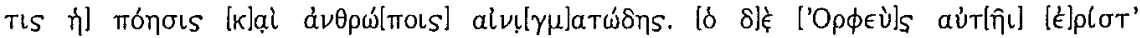

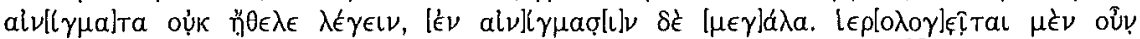

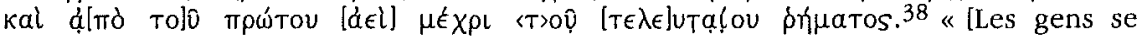
trompent en disant qu'Orphée n'a pas composé ${ }^{39}$ un hymne exprimant des mots sains et légitimes, car il a accompli avec son poème un acte religieux. Il n'est pas possible (pour eux) de dire l'interprétation des noms et ce qu'ils signifient. Car la poésie est quelque chose d'étrange pour les gens, comme une énigme. Or Orphée ne voulait pas dire des devinettes pour discuter, mais de grandes choses à travers des énigmes. Et, ce qui plus est, il y prononce un discours sacré dans toute son étendue, du premier mot au dernier ${ }^{40} »$.

Nous observons qu'il y insiste sur la légitimité du récit, même si quelques passages de la narration, tels que les actes incestueux ou de cruelle voracité, peuvent paraître abominables. Une exégèse " correcte », d'après laquelle le poème ne dirait pas ce qu'il dit littéralement, transformerait de telles actions en actions « légitimes " ${ }^{41}$. La phrase « il a accompli avec son poème un acte religieux» signifie probablement que celui-ci était employé dans la célébration d'un rituel orphique. Cette mention de la poésie comme une énigme, ainsi qu'une autre postérieure :

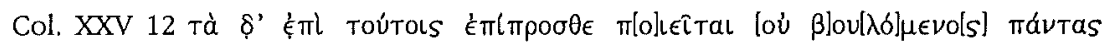
$\gamma(\nu \mid \omega ́) \sigma \kappa \xi ̨[l] v^{42}$, «Les mots qui suivent il les composa parce qu'il ne voulait pas qu'il fussent connus de tout le monde ».

anticipent Platon, Alcib. II, 147 b :

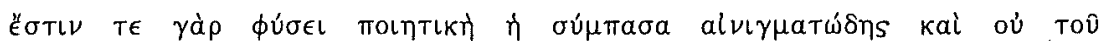

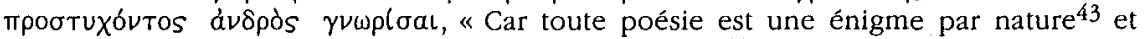
n'est pas faite pour que tout le monde la comprenne ${ }^{44}$.

Notons que le commentateur croit connaître le sens véritable des mots du poète, car il dit que la poésie est « comme une devinette pour les gens », et il

38 Tous les suppléments viennent de Tsantsanoglou (1997) 117 sq., sauf kal т[à] Janko (Kal[Tol] Ts.) et «T>ộ Janko. L'addition de (pour eux) dans la traduction est aussi de Janko.

39 Excepté cette première partie, qui est une reconstruction exempli gratia, je ne marquerai pas, dans la traduction, les crochets indiquant les parties qui constituent des suppléments au texte grec.

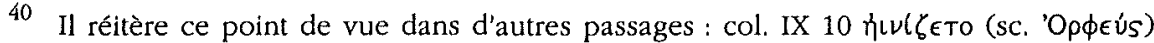

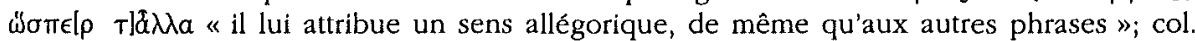

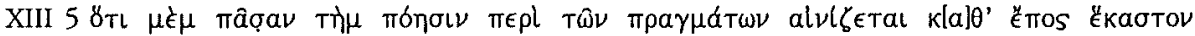

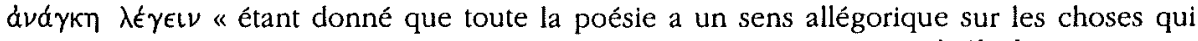
existent, il faudra la commenter mot à mot "; col. X 11, XVII 13 ạ alv $[\mathfrak{l} \zeta \in$ Tal « il dit de façon énigmatique ».

41 Comme le signale Tsantsanoglou (1997) 119.

42 Texte de $Z P E$ (1982).

43 Dans le sens qu'elle contient un sens caché qui doit être interprété.

44 Cf. Bernabé (1999a). 
est bien clair, comme il arrive souvent dans ce genre d'expressions, qu'il ne se sent pas inclus dans le générique « les gens». Par rapport à l'expression « des énigmes pour discuter», elle a probablement le sens technique d'« énigmes éristiques », qui étaient des devinettes destinées à devenir un pur exercice philosophique sans conséquence. Pour le commentateur, au contraire, les mots d'Orphée ont bien un sens qui peut et qui doit être interprété ${ }^{45}$.

Cette méthode du commentateur a conduit quelques spécialistes à considérer ce dernier comme peu fiable ${ }^{46}$. Il est certain qu'il nous oblige à adopter une attitude prudente à l'égard de ses interprétations, lors de l'analyse du poème, et que nous ne pouvons accepter qu'il attribue au poète des intentions qui apparaissent comme anachroniques au moment de la composition de l'Hymne. Mais il peut nous être utile, lors de la reconstitution du poème, car nous pouvons toujours saisir (parfois presque deviner), ne serait-ce qu'une petite partie de tout ce qu'il ne cite pas littéralement, dans ce qu'il commente. Et nous en avons bien besoin, parce que l'auteur, lui, lisait le poème entier, alors que ce n'est pas possible pour nous. Naturellement, notre méfiance à l'égard de sa manière de commenter ne doit pas être une raison pour mésestimer son témoignage sur le texte ${ }^{47}$, particulièrement en tout ce qui touche à l'ordre dans lequel il commente ses différents passages, car tout semble indiquer qu'il les cite en fonction de leur ordre d'apparition dans l'ouvrage original ${ }^{48}$.

\section{Le texte et le commentaire de la théogonie}

\subsection{Avis préalables}

Voyons maintenant comment reconstituer cette espèce de puzzle incomplet, dont les pièces seraient les vers ou les parties citées par le commentateur. Nous pouvons compter dans cette entreprise sur un autre élément de jugement: la Théogonie en 24 Rhapsodies (communément appelée les Rhapsodies), dont le sujet présente des points communs avec le récit contenu dans l'Hymne, bien que ce ne soit pas le même. En effet, les Rhapsodies sont un poème beaucoup plus long, ayant plus ou moins altéré les versions anciennes de la théogonie orphique. Et même si les arguments ex silentio ne constituent pas des preuves, il est bien clair que certains personnages de la théogonie rhapsodique ne sont même pas mentionnés dans les vers cités et qu'aucune allusion à ceux-ci n'a été faite par le commentateur. Je veux plus précisément dire que dans notre texte aucune mention n'est faite

45 Cf. Tsantsanoglou (1997) 122.

$46 C f, e . g$. West (1983) 88 « the commentator ..., who is in general the least trustworthy of guides ».

47 Cf. la défense qu'en fait Betegh (1999) au début du premier chapitre.

48 Cf. Betegh (1999) $\$ 1.4$, qui part d'une analyse de la manière dont les citations sont introduites dans le commentaire. 
du Temps ${ }^{49}$ ni de l'œuf cosmique, ni de Phanès, un monstre serpentin à quatre têtes, même pas sous la forme d'Éros ${ }^{50}$; pourtant, ces êtres remplissaient tous, dans les Rhapsodies, des rôles primordiaux. À mon avis, cette absence montrerait qu'aucun de ces êtres n'apparaissait dans le poème orphique objet du commentaire. J'exposerai plus tard les arguments sur lesquels j'appuie mon affirmation.

Je vais diviser mon analyse des fragments suivant les différents épisodes que j'ai reconstitués dans l'intrigue du poème. Le texte que nous présentons ici est celui de mon édition des fragments orphiques sous presse dans la Bibliotbeca Teuberiana, mais l'apparat critique sera ici extrêmement sélectif. Tout au long de cette analyse, les fragments littéraux du poème apparaîtront écrits en grec, en gros caractères, alors que les non littéraux seront représentés en petits caractères. Enfin, leur numérotation correspond aussi à celle que nous leur avons attribuée dans la future édition.

\subsection{Le proème}

Dans une partie très endommagée du papyrus apparaît une référence indirecte à un vers introductif, probablement le premier du poème. Nous pouvons le reconstituer ${ }^{51}$ parce que nous le retrouvons dans d'autres poèmes orphiques et qu'il est même devenu un archétype pour faire référence à des textes qui ne peuvent être compris que de quelques-uns ${ }^{52}$ :

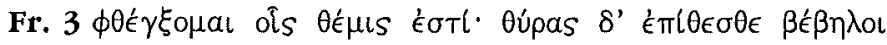

Je parlerai à ceux à qui cela est permis; fermez les portes vous autres, profanes.

Le poète manifeste ici qu'il ne s'adresse qu'aux initiés. Ceux qui ne le seraient pas sont sommés de fermer leurs portes, ce qui signifie qu'ils ne doivent pas lire l'hymne. Il ferait allusion ensuite au «plan » de l'ouvrage auquel appartiendrait probablement le vers suivant :

49 Cf. l'analyse de Betegh (1999) $₫ 2.2 .2 .1$

50 Tel que l'a signalé Betegh (1999) \$ 2.2.3.1, lorsque le commentateur se réfère dans la col. XXI à la conjonction physique des particules, il ne parle pas d'Éros mais d'Aphrodite, ce qui semble prouver qu'Éros n'était pas mentionné dans le poème.

51 Cf. col. VII 8 (les suppléments au texte, sauf avis contraire, sont de Tsantsanoglou)

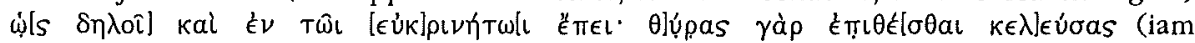

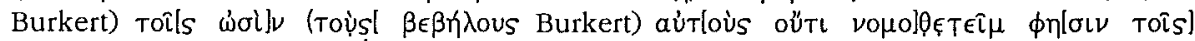

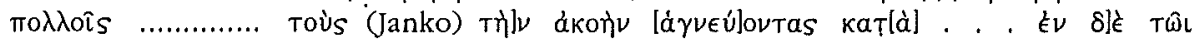

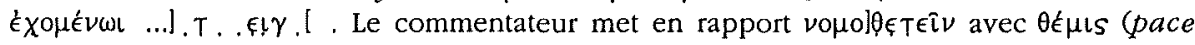
Tsantsanoglou [1997] 124 « why then is the text not quoted but only refered to as Eukк another poem that is being referred to and not the Orphic theogony ». Mais il se peut que

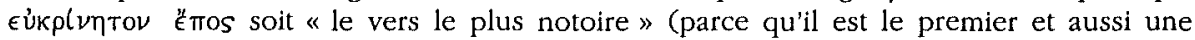
sorte de $\sigma \phi \rho a \gamma(s$ des poèmes orphiques, $c f$. Bernabé [1996b]) et kal signifie «même ». $C f$. aussi Casadesús (1995) 237 sq.; 480, Janko (1997) 67, (2001) 21, Parker (1995) 489.

$52 C f$. les détails et l'histoire du processus dans Bernabé (1996b). 


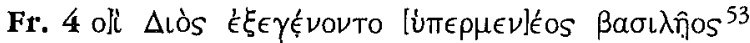

qui naquirent de Zeus, le monarque tout-puissant.

Le poète doit se référer ici aux dieux et aux êtres qui sont nés de Zeus, ce qui nous indique que le sujet central du poème est ce rôle démiurgique de Zeus, engendrant les dieux et les êtres qui configureront le monde.

\subsection{Zeus prend le pouvoir des cieux}

Le fr. 5 suivait fort probablement le vers antérieur, qui serait le dernier vers du proème ${ }^{54}$. Il semble que l'action commence au moment où Zeus reçoit le pouvoir de son père, Kronos.

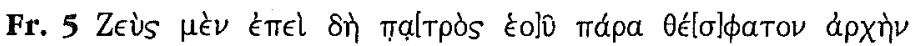

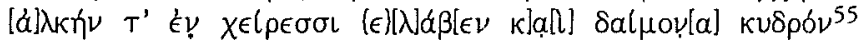

Zeus, lorsqu'il reçut de son père le pouvoir décerné par les dieux et qu'il prit dans ses mains la force, et l'illustre divinité...

Depuis le début, le poète insiste sur les aspects justes du règne de Zeus. Sa souveraineté est légitime parce que «décernée par les dieux». Le deuxième vers peut être interprété de deux manières; a) ou bien " l'illustre divinité " serait l'objet direct d'un verbe que apparaîtrait dans le vers suivant, non cité par le commentateur, ce qui semble peu probable, ou b) il s'agit d'un hendiadys signifiant « il prit dans ses mains le pouvoir et la force de l'illustre divinité ». Quoi qu'il en soit, l'expression «il prit dans ses mains» est fréquemment employée dans la littérature théogonique lorsqu'on veut faire référence à des transmissions de pouvoir ${ }^{56}$.

On peut se demander qui était cette «illustre divinité ». D'après West ${ }^{57}$ il s'agirait de Phanès Protogonos, l'un des personnages des Rhapsodies. Et, pour appuyer son idée, il force substantiellement le texte ${ }^{58} . \AA$ mon avis, et

53 Col. VIII 2. Les suppléments au texte sont déjà présents dans $Z P E$ (1982). West (1983) 114 restitue ici trois vers exempli gratia.

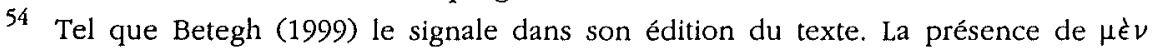
emphaticum au premier vers à amené Calame (1967) 67 n. 3 à penser, avec raison, qu'il s'agissait du début de la narration généalogique.

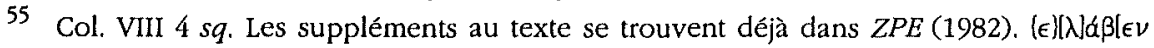
est une correction metri causa.

56 Cf. fr. 102 Kern (et le fr. 101 Kern avec le verbe $\theta \hat{\kappa} \kappa \epsilon)$ et Casadesús (1995) 247.

57

West (1983) 84ss; 114 .

58. Concrètement il transpose le vers 2 en continuation du fr. 7 et lit ensuite le

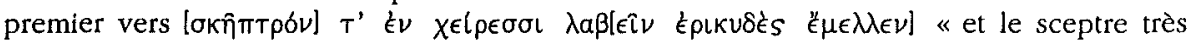
glorieux, il allait le prendre dans ses mains " (avec l'accord de Rusten [1985] 126). Cf. aussi l'opinion plus prudente de Casadesús (1995) 247. Quant à Tsantsanoglou, il considère, dans un courrier, que le $1^{\text {er }}$ vers et le fr. 7 du texte ne sont que deux versions du 
étant donné que je ne crois pas qu'il y ait la moindre allusion à Phanès dans le poème (un aspect sur lequel nous reviendrons), il serait plus pertinent de

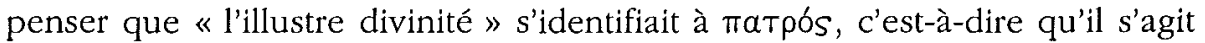
de Kronos, et que la phrase fait référence au moment où Zeus le chasse du pouvoir et s'empare de son royaume. En dépossédant son père du trône, il le tient dans ses mains et c'est ainsi qu'il conquiert la souveraineté (le gouvernement du ciel) et sa force (en tant que dieu suprême).

\subsection{Les prophéties de la Nuit et de Kronos}

Après s'être emparé du pouvoir, Zeus se rend dans la grotte de la Nuit, laquelle lui indique, de manière prophétique, comment il devra exercer son pouvoir $^{59}$ :

Fr. 6 Z $\varepsilon \dot{v} s \mu \dot{v} \nu$...

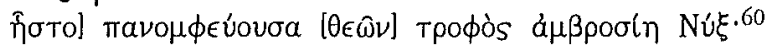

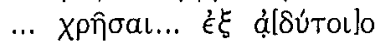

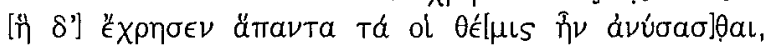

ws du

Et Zeus (arriva à la grotte, où) ${ }^{61}$

siégeait la Nuit, connaisseuse de tous les oracles, immortelle nourrice des dieux

... prédire du plus caché,

Elle lui prédit tout ce qu'il lui serait licite d'emporter:

comment il occuperait le beau trône de l'Olympe enneigé.

Le rôle de vaticinatrice est caractéristique de cette déesse dans les théogonies orphiques ${ }^{62}$, sans doute par son caractère primordial qui lui permet de tout savoir. L'action de Zeus y est à nouveau qualifiée de légitime, car l'exercice de sa souveraineté sur les dieux est défini comme « tout ce qu'il lui serait licite de remporter ». Et Kronos indique également à Zeus comment poursuivre son œuvre créatrice :

même vers, tout en marquant sa préférence pour le fr. 7 comme $1^{\text {er }}$ vers du fragment.

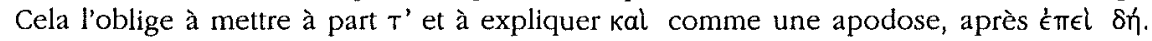

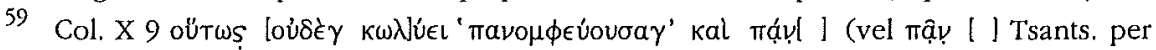

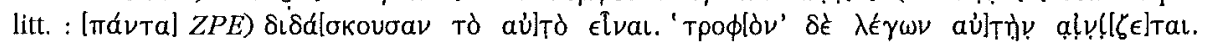

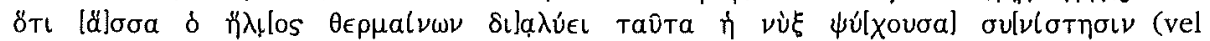

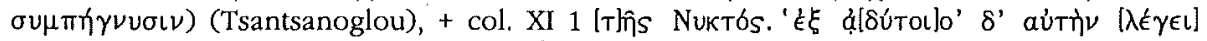

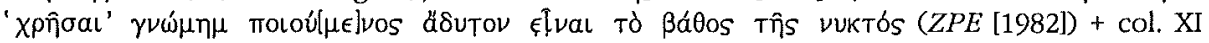
10 (v. 4 suppl. Tsantsanoglou) + col. XII 2 (v. 5 suppl. Tsantsanoglou).

60 Sur la figure de la Nuit dans les cosmogonies, orphiques ou non (bien qu'elle ne se soit pas occupé de celle de Derveni), $c f$. Ramnoux (1986); sur la Nuit, dans la Théogonie de Derveni, $c f$. Tortorelli (1985) (1991), et dans les Rhapsodies $c f$. Bernabé (1998a).

61 Une reconstitution conjecturale très vraisemblable, du fait que la Nuit habite toujours dans une grotte dans les théogonies orphiques.

62 Cf. Bernabé (1998a). 


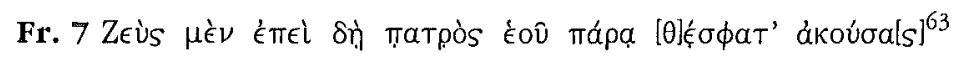

Zeus, après avoir entendu les prédictions de son père...

Le fait qu'il y ait une double prédiction, celle de la Nuit et celle de Kronos, semble déconcerter Rusten ${ }^{64}$, lequel, s'appuyant sur la ressemblance du début de ce vers avec le premier vers du fr. 5, postule que le commentateur "a préféré (ou inventé) une lecture alternative ». Une si curieuse appréciation a été justement critiquée ${ }^{65}$. En effet, ni la répétition d'une grande partie d'un vers, dans ce type de poésie essentiellement formulaire, ni le fait que Zeus reçoive des prophéties de son père, en plus de celles de la Nuit, ne devraient surprendre, d'autant plus que dans les Rhapsodies, en plus des prophéties de la Nuit, on parle aussi de celles de Kronos, cf. Procl. in Plat. Cratyl. 27, 21 Pasquali (fr. $155 \mathrm{Kern})$ :

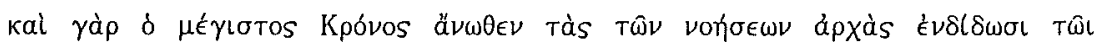

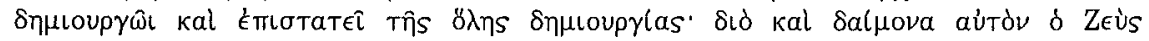

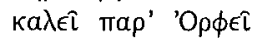

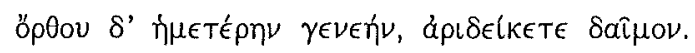

Et Kronos, du haut de sa grandeur, livre au démiurge les principes des intellections et dirige toute la démiurgie. Voilà pourquoi Zeus l'appelle " démon " dans Orphée :

Conduis mon lignage par le droit chemin, illustre démon.

Tel que l'indique Casadesús ${ }^{66}$ « la nuit prédit, mais c'est le père qui offre à Zeus les principes démiurgiques ».

\subsection{L'exécution des conseils de Kronos et de la Nuit}

Le conseil le plus important de Kronos et/ou de la Nuit est celui que Zeus exécute dans le fragment 8 et qui consiste à avaler quelque chose ou quelqu'un.

Pour interpréter ce passage nous devons l'analyser en parallèle avec celui du début du fr. 12 , qui se réfère à la même circonstance. Et nous accompagnons l'un et l'autre des commentaires de l'auteur anonyme du texte, dont nous avons besoin pour notre reconstitution:

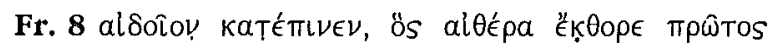

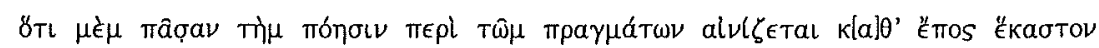

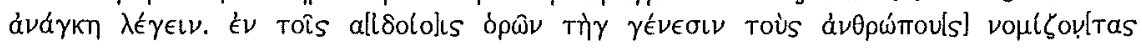

63 Col. XIII 1. Les suppléments au texte se trouvent déjà dans ZPE (1982).

64 Rusten (1985) 125 sq.

65 Edwards (1991) 204 sq., Casadesús (1995) 294 sq.

66 Casadesús (1995) 296. 


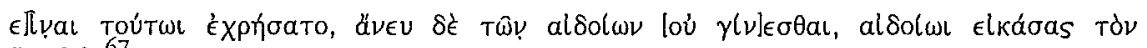
$\eta \lambda \lambda \operatorname{co}^{2}{ }^{6}{ }^{67}$

Il avala le phallus (ou le vénérable)... ${ }^{68}$

Étant donné que tout le poème a un sens allégorique sur les choses qui existent, il devient nécessaire de le commenter mot à mot. Il (Orphée) employa ce mot (aidoion) car il vit que les hommes croyaient que la génération résidait dans les organes génitaux et que sans organes génitaux il n'y avait aucune possibilité de génération, aussi compara-t-il le soleil au phallus.

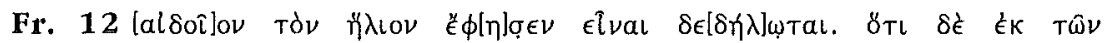

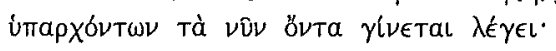

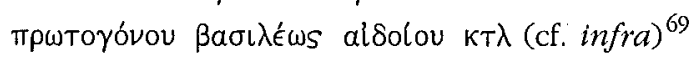

Il demeure bien clair qu'il appela le soleil " phallus " et qu'il dit que les choses qui existent aujourd'hui proviennent de celles qui existaient auparavant ;

du phallus du (ou du vénérable) roi aîné...

Les plus grandes difficultés d'interprétation de ce passage (qui concernent d'ailleurs la compréhension de toute la Théogonie) dérivent de deux ambiguités, aggravées par le caractère incomplet du texte qui nous est par-

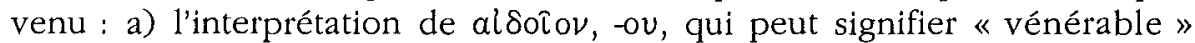
(adjectif) et «phallus » (substantif) ${ }^{70}$, et b) l'identification de cet aîné. J'avance que, à mon avis, alsotov signifie " phallus » et que l'aîné est le Ciel. Mais analysons les différents éléments soumis à discussion.

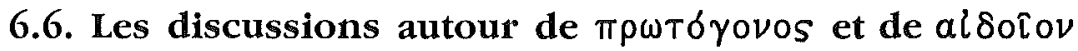

$\grave{A}$ propos de alsô̂ov, quelques auteurs ${ }^{71}$ ont affirmé qu'il s'agissait du phallus du Ciel que Kronos avait préalablement amputé. Le commentateur construit sans aucun doute son commentaire comme si le poète avait donné à aldotov sa valeur substantive, c'est-à-dire, "il avạla son phallus ». Tout choquant que ce motif thématique puisse paraître, on le retrouve déjà dans un précédent oriental. Il s'agit d'un mythe hourrite que nous connaissons dans une version hittite ${ }^{72}$, et dont les ressemblances avec le mythe hésiodique ont été soulignées à plusieurs reprises. Dans ce mythe, Anou, le dieu

67 Col. XIII 4 sq. Toutes les reconstitutions du texte se trouvent déjà dans $Z P E$ (1982).

68 Pour ne pas trop compliquer la discussion sur cet aspect, je remets à plus tard l'interprétation de la proposition relative.

69 Col. XVI 1. Toutes les reconstitutions du texte se trouvent déjà dans $Z P E$ (1982).

70 Déjà attesté au singulier avec ce sens-là, par ex., chez Hérodote (II, 30 et 48).

71 Burkert (1980) 32, (1987) 22, (1998) 389 et n. 14, Kirk - Raven - Schofield (1983) 32, Graf (1985) 588, Bernabé (1989) 169; Janko (2001) 24 et n. 124. Cette interprétation est aussi celle de Betegh (1999) après son analyse des arguments dans $₫ 1.5$.

72 Qu'on a appelé le Règne aux Cieux ou Théogonie, cf. Laroche (1965) 153 sq., Bernabé (1987) 139 sq. 
du Ciel, est émasculé par la morsure de Koumarbi (l'homologue hourrite de Kronos), de telle sorte que ce dernier, après avoir avalé les organes génitaux du Ciel, se retrouve « enceint » de plusieurs dieux. À son tour Zeus, dans la théogonie orphique, aurait avalé le phallus du Ciel, ce qui aurait donné lieu à sa grossesse du cosmos.

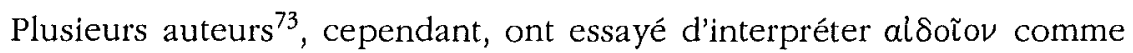
un adjectif, qualifiant un personnage qui aurait été avalé tout entier par Zeus. Et ce personnage ne serait autre que Phanès, tout comme dans les Rhapsodies. Ceci impliquerait, d'une part, que Phanès - dont il n'y a nulle autre trace dans les fragments conservés du papyrus - serait l'un des personnages de notre hymne et qu'il faudrait dès lors le localiser dans la généalogie qui y est mentionnée. Et, de l'autre, que le commentateur, qui avait sous les yeux le poème entier, l'aurait compris moins bien que nous, qui ne l'avons pas. Une solution moyenne visant à expliquer cette interprétation

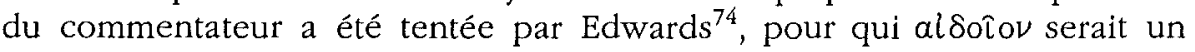
adjectif dans notre texte, mais le commentateur « detects in the Orphic poem a comparison which transpires through etymology, but only such a comparison as bequeathes a deeper riddle to the wise ".

Or, on peut voir que, dans ce passage, le commentateur ne nous dit pas que le poète compare quelqu'un au soleil en l'appelant «vénérable » (cela n'a pas de sens). La situation initiale en est que le commentateur se trouve devant une expression signifiant quelque chose d'apparent (pour les gens) et quelque chose de caché ou d'énigmatique (pour lui), car le mot al soîov "phallus » serait peu convenable, dans son sens littéral, d'après sa propre considération du langage d'Orphée. Il n'en irait pas ainsi s'il signifiait "vénérable ${ }^{75}$. En fonction de cet indiscutable principe de base, nous comprenons bien que si le commentateur dit que aíốov (phallus) se réfère énigmatiquement au Soleil (véritable principe vivifiant de la réalité, tout comme les organes génitaux, qui produisent la génération des choses) c'était parce qu'il avait sous les yeux un texte que non seulement lui-même mais tout individu possédant une compréhension correcte du grec comprenait comme « le phallus du Ciel ». Il eût été, pour lui, absurde de gloser en ces termes un alsoîo $\nu$ placé dans un contexte où il ne pouvait rien signifier d'autre que "vénérable », se rapportant à un personnage quelconque. De son côté, l'interprétation d'Edwards demanderait deux changements de niveau de sens : a) comprendre que "vénérable Ciel » doit être interprété comme «le phallus du Ciel » et b) qu'il en est ainsi parce que cette expression se réfère au Soleil. Une démarche trop compliquée, même pour le commentateur de Derveni. Nous devons donc considérer l'interprétation d'Edwards comme ingénieuse mais erronée.

73 West (1983) 85, Rusten (1984) 334, (1985) 125, Parker (1995) 490 sq.

74 Edwards (1991) 205 sq.

75 Tel que le signale Betegh (1999) $₫ 1.5$ fort à propos. 
Nous pouvons encore citer un autre passage curieux qui, sous cette lumière, prend une signification nouvelle ${ }^{76}$. C'est un texte de Diogène Laerce (Prooem., 1, 5):

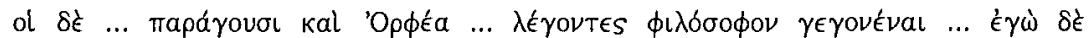

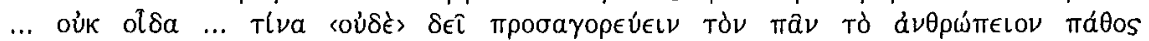

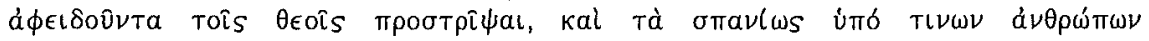

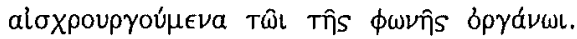

D'autres nous présentent Orphée en disant que ce fut un philosophe. Mais je ne sais comment appeler quelqu'un qui attribue immodérément aux dieux toute sorte de passions humaines et même des actes honteux (exécutés) avec l'organe de la parole qui sont rarement pratiqués par les hommes.

Nous n'avons pas d'autre texte attribué à Orphée où les dieux pratiquent la fellatio, aussi ne serait-il pas invraisemblable que Diogène Laerce fasse précisément référence à ce passage.

En ce qui concerne l'identification de cet «aîné », le fait que dans les Rhapsodies Phanès soit appelé l'aîné a amené plusieurs auteurs à considérer que dans cette théogonie l'adjectif aîné faisait également référence à Phanès ${ }^{77}$. Or, « aîné » est bien une épithète qu'on peut appliquer à n'importe quel premier né. Et, jusqu'ici, dans notre reconstitution, le premier né serait le Ciel, étant donné que sa mère, la Nuit, on la suppose éternelle. Évidemment, le commentateur ne fait dans son texte aucune allusion à rien qui puisse nous rappeler les aspects monstrueux de Phanès, dont on dit dans les Rhapsodies qu'il possède quatre têtes de différents animaux, qu'il est aussi ailé et serpentin en même temps, avec les organes sexuels placés dans le dos. Il est impensable que le commentateur, ayant rencontré un tel monstre dans l'hymne orphique, ait gardé dans son commentaire, à son sujet, le silence le plus absolu. D'ailleurs Phanès est un personnage qui s'incorpore très tard aux textes littéraires et plus tardivement encore à l'iconographie ${ }^{78}$, toujours associé à une cosmogonie initiée par un Temps primordial personnifié, qui montre, lui aussi, des traits monstrueux. Et nous avons déjà souligné ici qu'il n'y a pas la moindre trace du Temps dans notre poème ${ }^{79}$.

Mais le véritable problème à cet égard consiste à savoir quelle aurait été la place de Phanès, dans la lignée des successions, au cas où il apparaîtrait réellement dans le poème. J'essaierai de montrer qu'il n'y a aucune possibilité qu'il y prenne place. Le témoignage du fr. 10 nous semble nettement explicite à ce propos :

\footnotetext{
76 Passage mentionné par Burkert (1999) $81 \mathrm{sq}$.

77 West (1983) 85, Rusten (1984) 334, (1985) 125, Parker (1995) $490 \mathrm{sq}$.

78 Cf. Turcan (1994) 363 sq., où nous voyons qu'il n'y a pas de représentations de ce personnage jusqu'à une époque très tardive ( $\mathrm{II}^{\mathrm{e}}$ s. av. J.-C.).

79 Cf. la brillante argumentation de Betegh (1999) $\$ 1.5$.
} 


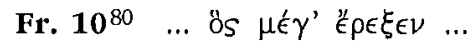

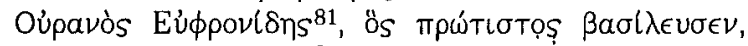

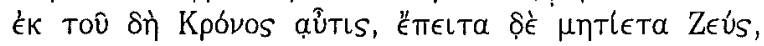

(Kronos) qui fit quelque chose de terrible...

Ciel, l'enfant de la Nuit, qui fut le tout premier roi.

Il y est dit, en effet, que le Ciel est Euphronide, c'est-à-dire, le fils de la Nuit $^{82}$, et qu'il a été le tout premier roi; ce qui implique, en toute logique, que si la Nuit est bien antérieure au Ciel (car elle est sa mère), elle n'a pas régné. Curieusement Rusten ${ }^{83}$ ne considère pas que ce vers est incompatible avec la possibilité que d'autres aient régné avant le Ciel, et il cite l'emploi de $\pi \rho \omega$ tos

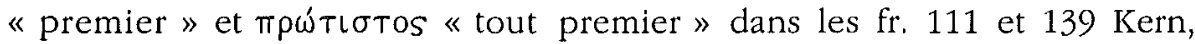
appartenant tous les deux aux Rhapsodies, à l'appui d'un usage hypothétique en fonction duquel ces mots ne signifieraient pas tout à fait « le premier ». Or ces rapprochements sont inadéquats. Dans le fr. $111 \mathrm{Kern}$, nous lisons au sujet du Ciel :

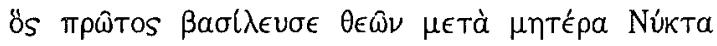

c'est lui qui fut le premier des dieux qui régna, après sa mère, lâ Nuit.

Il est vrai qu'on y dit que « le Ciel fut le premier des dieux qui régna », bien que son règne se soit produit après celui d'autres dieux, mais le vers se

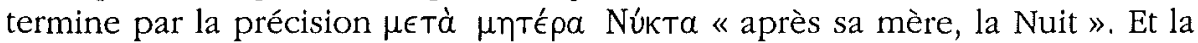
Nuit constitue en quelque sorte dans les Rhapsodies un point d'inflexion dans la génération des dieux, en y délimitant un avant et un après. Et ce serait justement le Ciel que inaugure la deuxième période. Il n'est donc pas question ici, au contraire de notre passage, qu'il ait été «le premier roi » en termes absolus.

Le deuxième exemple, le fr. 139 Kern, fait mention de Kronos en disant :

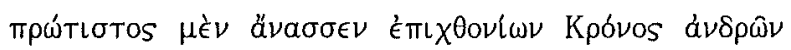

ce fut le tout premier des dieux qui régna sur les hommes qui habitent sur la terre.

L'important de ce passage, c'est que, comme les hommes n'existaient pas avant le règne de Kronos, on y souligne précisément que ce fut le premier dieu à régner non seulement «sur les dieux » mais aussi « sur les hommes».

80 Col. XIV 5 s. + Col. XV 6.

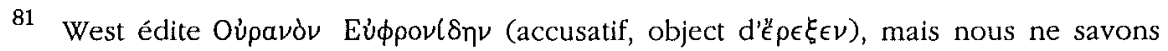

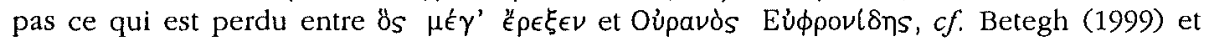
autrement Rusten (1985) 135 n. 30.

82 Sur Eủ $\phi$ povl( $\eta$ s cf. Dunand (1973) III 110 sq., Corsten ad Inschr. Kios 21, 7 p. 100, Tortorelli (1985) (1991).

83 Rusten (1985) 135 n. 31. 
L'Hymne cité dans le Papyrus de Derveni ne présente aucune restriction de ce genre, aussi l'expression citée doit-elle bien signifier qu'aucun dieu n'a régné avant le Ciel, et cela est incompatible avec la présence de Phanès dans ce poème. Il est par contre vraisemblable que la Nuit, mère du Ciel, ait été dans cette version un être primordial qui n'est pas parvenu à exercer le pouvoir. D'autres traits intéressants du poème viennent à l'appui de cette idée et la rendent encore plus vraisemblable :

a) L'épithète « nourrice des dieux » (fr. 6) attribuée à la Nuit, de même que ses pouvoirs prophétiques, dont nous avons déjà parlé, sont caractéristiques d'une divinité primordiale.

b) Dans un passage d'Aristote (Métaph., 1071b 26) apparaît probablement une allusion à Orphée, comme l'un des théologiens qui font dériver la

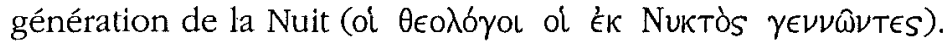

c) Dans un autre passage du même ouvrage (Métaph., 1091b 4), le Stagirite nous parle des «poètes archaïques... en tant qu'ils disent que ce ne sont pas les êtres premiers - comme la Nuit, le Ciel, Chaos ou l'Océan - qui ont

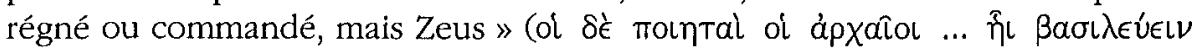

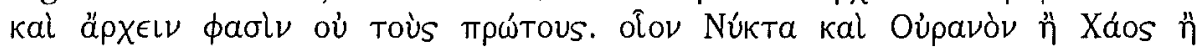

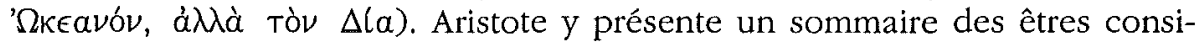
dérés comme les premiers dans plusieurs théogonies anciennes, parmi lesquelles nous reconnaissons celle d'Hésiode (Théog., 116 sq. : le premier être aurait été le Chaos) et celle d'Homère (Il. XIV, 200, 246: les parents de la création sont Okéanos et Téthys), de telle manière que celle qui commençait par la Nuit était probablement celle d'Orphée, bien connue à cette époque-là et qui appartient clairement à la même tradition que celle qui est commentée dans le Papyrus de Derveni ${ }^{84}$. Tout parait clair jusqu'ici. Mais nous trouvons une note dissonante dans le fragment qui suit :

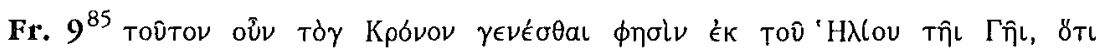

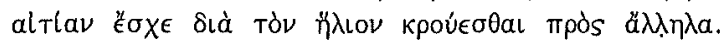

C'est ainsi qu'il (Orphée) dit que ce Kronos naît du Soleil pour la Terre, parce que ce fut à cause de lui, à travers le soleil, que les choses se sont heurtées les unes aux autres.

La deuxième partie du fragment répond au goût étymologisant du commentateur, associé à sa méthode allégorique, qui l'amène à expliquer Koóvos à partir du verbe kpoúw «se heurter ${ }^{86}$, plus concrètement, comme dérivé de

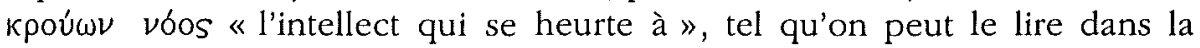
colonne XIV 7 :

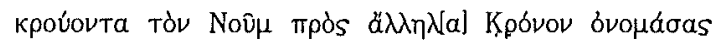


en appelant l'intellect, qui fait les choses se heurter les unes aux autres, du nom de Kronos.

Cette explication étymologique, certainement originale, permet au commentateur de remplacer allégoriquement la généalogie traditionnelle, Ciel Kronos, par une espèce d'interprétation atomiste où prennent part le Soleil, en tant que source de chaleur, et toute une série de particules s'entrechoquant mécaniquement à la manière de la théorie atomiste, encore que l'intervention de vous la rapproche plutôt d'une explication similaire à celle d'Anaxagore $^{87}$.

La première partie du fragment, où se trouve l'affirmation que Kronos naît du Soleil et de la Terre, apparaît encore plus problématique. Rusten ${ }^{88}$ qui soutenait la présence de Phanès dans le poème, postule ici que le commentateur assimile le Ciel au Soleil, une affirmation qui est naturellement incompatible avec son explication première. West ${ }^{89}$ remarque avec surprise que « so it looks as if the poem, as the commentator read it, represented the swallowed god ... as the father of Kronos by Ge, » pour observer ensuite que dans les Rhapsodies et chez Hésiode le père de Kronos est le Ciel.

Cette contradiction ne serait qu'apparente, pourvu que l'on ne prétende pas inclure Phanès dans la Théogonie de Derveni. Il est évident que, dans ce passage, le commentateur cite le Soleil à la place du Ciel comme le père de Kronos alors que dans la séquence de la transmission du pouvoir (fr. 10, 2-3), le poète nous présentait la suite habituelle Ciel - Kronos - Zeus. Tout ceci nous conduit à comprendre, soit que le commentateur identifie le Ciel au Soleil dans son texte, soit qu'il croit, plus particulièrement, que le Soleil est le phallus du Ciel. Et il est probable que c'est cette dernière qui est l'interprétation correcte, d'autant plus que les références analysées plus haut semblent le confirmer :

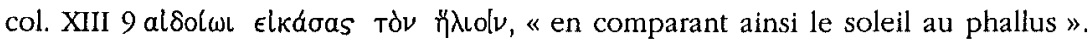

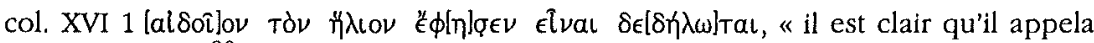
'phallus' le soleil $)^{90}$

Tel que Betegh ${ }^{91}$ le signale, « $\phi \eta \sigma(\nu$ here (fr. 9) is not to be taken as introducing a verbatim quotation, but as accompanying an interpretative paraphrase (...) Kronos should be understood as the Mind that clashes things to each other, and this Kronos -i.e. Kronos understood in this way- is born

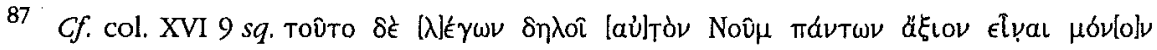
ŁovTa.

88 Rusten (1985) 136, cf. Edwards (1991) 207s et, pour une autre interprétation, Casadesús (1995) 315 sq.

89 West (1983) 88.

90 Les doutes de Calame (1991) 233, cf. (1997) 69 n. 6, sont ainsi dissipés, bien que Tsantsanoglou, dans une lettre qu'il m'adresse, pense que ceci remet en question tout le système physique de l'auteur du commentaire.

91 Betegh (1999) \$1.5. 
from the sun, since it is the heat of the sun which causes the things collide with each other ».

Bref, après une analyse d'ensemble de tous les textes significatifs du poème, la suite événémentielle suivante semble s'imposer : c'est le Ciel qui fut le premier né, car sa mère, la Nuit, était un être primordial et donc, non $n^{-92}$. Lorsque le Ciel fut émasculé par Kronos, son phallus resta dans l'espace. Cette circonstance, unie au caractère vivifiant et générateur de ce membre, conduit l'interprète à le considérer comme une métaphore du Soleil. Finalement Zeus, suivant les conseils de la Nuit et de son père, avalera le phallus du Ciel, ce qui produira sa fécondité créatrice ultérieure. Il est donc impossible de trouver une place pour Phanès dans le poème sans que cela ne contredise tout le reste.

\subsection{L'éjaculation de l'éther}

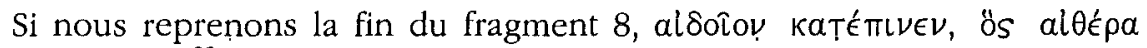

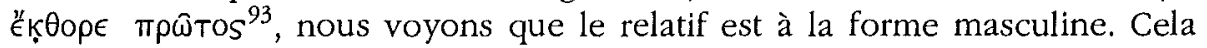
signifie que son antécédent ne peut être le mot neutre alsotov «phallus ", aussi nous faut-il supposer l'existence, dans le vers antérieur, d'un nom

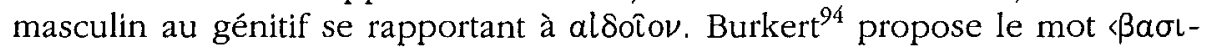

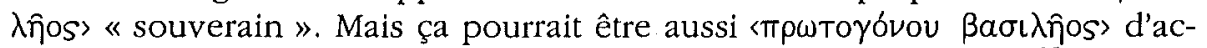
cord avec le fr. 12 . Et il est également possible d'y lire, avec Betegh ${ }^{95}$ Oúpavoû

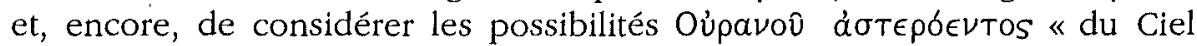

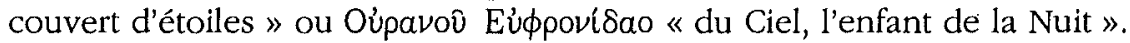

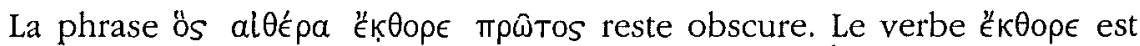
normalement employé pour se référer à un dieu qui naitt ${ }^{96}$, mais si on donnait ici cette valeur au verbe, al $\theta e ́ p a$ nous resterait très difficile à comprendre, car il faudrait l'interpréter, soit comme un accusatif de direction ${ }^{97}$ « celui qui fut le premier qui naquit à l'éther », soit comme un accusatif marquant la provenance $^{98}$ « celui qui fut le premier qui naquit de l'éther ». Dans les deux cas

92 La Terre devait y être mentionnée ou au moins considérée implicitement comme la mère de Kronos.

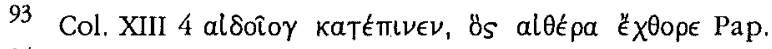

94 Burkert (1999) 81.

95 Betegh (1999) $\$ 1.5$.

96 Cf. Hymn. Merc., 20; Call., Hymn. 4, 255; Hymn. Curet. in Powell, Coll. Alex. p. 161, etc. Voir la note de Pfeiffer à Callim., fr. 43, 123; Rusten (1984) 334, (1985) 125 n. 9.

97 Comme le soutient Rusten (1985) $125 \mathrm{n} .9$ qui met le texte en parallèle avec

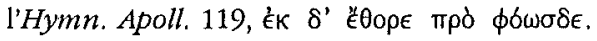

98 Tel que Edwards (1991) 207 le propose (suivi par Tsantsanoglou par lettre), en

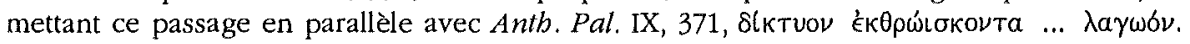


l'éther était déjà né ${ }^{99}$, et même dans le deuxième on pourrait considérer qu'il est en quelque sorte le progéniteur du Ciel, ou sinon une espèce de « matière » à l'intérieur de laquelle le Ciel se serait formé. Edwards ${ }^{100}$ observe à son tour que le mot ěk $\theta 0 \rho \epsilon$ semble connoter dans les poètes orphiques " jaillir d'une source ». Mais cette source peut difficilement être l'éther.

La proposition de Burkert ${ }^{101}$, d'après laquelle le Ciel aurait produit par éjaculation l'éther, nous semble plus sensée. C'est une interprétation qui s'appuie sur le commentaire de la colonne XIV 1 sq. où nous lisons :

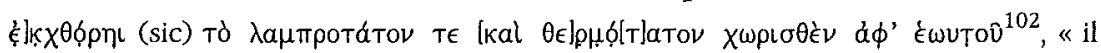
éjacula ce qu'il avait de plus brillant et de plus chaud, s'étant séparé de lui-même. »

Notons ici que ఢ̧] $]_{k} \chi \phi o ́ p \eta \iota$ est également construit avec un accusatif complément d'objet direct. Le commentateur aurait donc interprété l'éjaculation de l'éther dans le sens où celui-ci serait né par ségrégation de la partie brillante et chaude du Ciel.

Répétons maintenant le fragment 8 avec sa traduction :

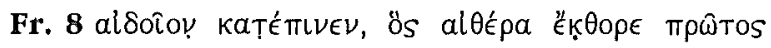

il dévora le phallus (du Ciel), qui avait préalablement éjaculé l'éther.

Cette démarche de Zeus va donner naissance non seulement à un nouveau règne divin mais aussi à une nouvelle étape cosmique. C'est en ceci qu'Orphée s'écarte complètement d'Hésiode; en effet, tandis que chez Hésiode le cosmos s'était organisé une fois pour toutes avant que les dieux ne commencent à lutter pour le pouvoir, dans le poème orphique le monde s'organise (ou, mieux, se réorganise) ensuite, lorsque le dernier des rois divins prend le pouvoir et anéantit toute opposition contre lui ${ }^{103}$.

\subsection{Les antécédents de l'histoire : la Nuit - Ciel - Kronos}

Notre argumentation nous a fait abandonner momentanément le fil narratif du poème. Il est temps de le reprendre. Après avoir fait mention de Zeus dévorant le phallus du Ciel (fr. 8), le poète tourne son regard vers les antécédents de l'histoire, bien que ce soit de manière très sommaire :

Fr. 10 ... ös $\mu \epsilon ́ \gamma^{\prime}$ "̈ $\rho \epsilon \xi \in \nu$

99 C'est pour cela que Betegh (1999) $\$ 2.2 .2 .1$ considère que l'Éther appartient à la première génération de dieux et que " can be taken not just as the male partner of Night, but also as the 'bright' counterpart of the 'dark' goddess. "

100 Edwards (1991) 206.

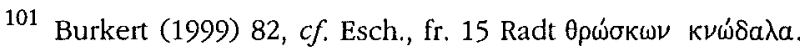

102 Tò Rusten; Tòv papyrus ( $c f . \chi \omega p\llcorner\sigma \theta \varepsilon \dot{\nu}$, qui est nettement un neutre). Les suppléments au texte sont de Tsantsanoglou. Sur la traduction, $c f$. Lévêque (1997) 238.

103 C'est ce qui se passe aussi dans le poème babylonien connu comme Enuma Elish, où Marduk ordonne le monde après s'être débarrassé de ses adversaires. 


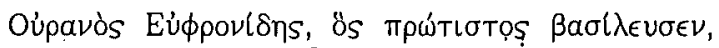

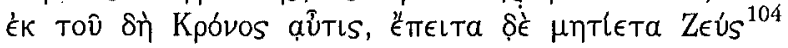

(Kronos) qui fit quelque chose de terrible...

Le Ciel, fils de la Nuit, qui fut le tout premier qui régna,

et de celui-ci, à son tour, Kronos, et ensuite Zeus le rusé.

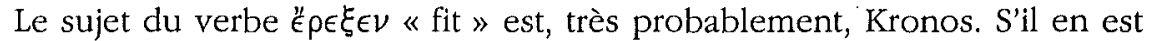
ainsi, et à la lumière du récit hésiodique (et des références pratiquement unanimes au mythe dans toute la tradition postérieure, y compris les orphiques), cette action «terrible » est l'émasculation ${ }^{105}$ du dieu, bien que le commentateur, qui prétend donner une interprétation « convenable » et " philosophique » du passage, se soit limité à indiquer ${ }^{106}$ que

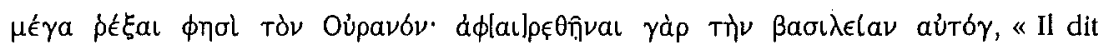
qu'il fit quelque chose de terrible au Ciel' parce qu'il lui extorqua la souveraineté »,

ce qui suppose, à mon avis, un clair indice que la mutilation perpétrée par Kronos était simplement indiquée, de façon très sommaire, dans le poème. Autrement l'interprétation « adoucie » serait déplacée.

Nous voyons donc que, à l'exception de l'inclusion de la Nuit dans la généalogie, comme la mère primordiale, le reste de l'histoire racontée dans la théogonie orphique répète, dans ses grandes lignes, celle d'Hésiode, bien que ce soit de façon très sommaire : Kronos mutile le Ciel et le chasse du pouvoir, pour être, à son tour, détrôné par Zeus. Et étant donné que, un peu plus tard (fr. 18), le texte nous parle de la mère de Zeus, il faudrait supposer aussi la présence dans le texte de la sœur et compagne de Kronos, Rheia, encore que, une fois de plus, le poète aurait pu omettre cette circonstance, en la considérant comme un fait acquis.

Le papyrus est particulièrement endommagé à cet endroit précis, de telle manière que, bien qu'une citation de trois vers y paraisse suivre, nous n'avons pu reconstruire que le premier de ces vers, lequel devait suivre le $\mathrm{fr}$. 10 , sans solution de continuité.

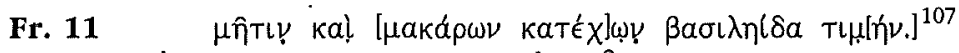

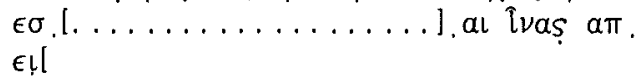

possesseur de la ruse et de la dignité royale des bienheureux ... les nerfs...

104 Cf. n. 81.

$105 C f$. West (1983) 86 y 114, qui en reconstruit exempli gratia deux autres vers.

106 Col. XIV 8 sq.

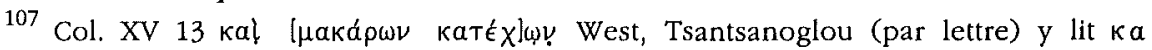
. [. ........... ] $]_{\xi} \mu$ et ajoute : «but I do not exclude altogether $\omega$ ». Le participe est plus vraisemblable ici, si l'on considère que ce vers venait très probablement, à mon avis,

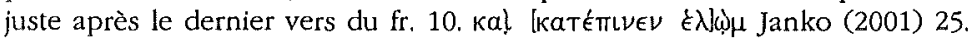


Je traduis par « ruse » le mot $\mu \hat{\eta} T \iota s$, qui renferme un concept complexe, impliquant des attitudes mentales et des comportements intellectuels qui combinent la sagacité, la prévoyance, la souplesse d'esprit et la simulation et bien des aspects encore, comme Detienne et Vernant ${ }^{108}$ l'ont souligné. Ce mot peut, d'ailleurs, apparaître dans les textes personnifié, comme il l'a été chez Hésiode, lequel nous le présente dans Théog. $886 \mathrm{sq}$. au féminin, comme l'épouse de Zeus, que le dieu aurait avalée alors qu'elle était enceinte, pour éviter d'être détrôné par l'enfant qu'elle mettrait au monde ( $c f$. v. 358). Dans la poésie orphique postérieure, Mêtis, au masculin, est identifié avec Éros-Phanès-Protogonos, ce qui a fait que plusieurs auteurs ont assimilé cette Mêtis personnifiée à Phanès ${ }^{109}$; or, comme j'ai déjà argumenté ici, il semble impossible de situer Phanès dans notre ouvrage. Mais ce ne serait pas, non plus, possible d'y placer la Mêtis hésiodique, personnage féminin. Par contre, l'interprétation de $\mu \hat{\imath} \tau \iota \nu$ comme un nom commun serait parfaitement admissible ${ }^{110}$, du fait que le poète orphique aurait réinterprété en le rationalisant le passage hésiodique où la déesse Mêtis est dévorée par Zeus, dans le but d'expliquer l'étymologie de l'épithète $\mu \eta \tau \imath \in T \alpha$ (fr. 10, 3) attribuée à Zeus (fr. 10, 3) et aussi $\mu$ nбaтo (fr. 16, 1-2), du fait que Zeus aurait intégré (en avalant le phallus du Ciel) la ruse et la dignité royale des bienheureux, c'est-àdire, le pouvoir et les ressources nécessaires pour réorganiser la création.

Nous ne pouvons rien dire du morceau qui suit, où l'on n'arrive à lire que le mot lvas "nerfs", dont la justification dans ce contexte me semble absolument énigmatique. Il est vrai que, plus loin dans le texte, on parle des « nerfs de l'Achéloos », mais cette référence ne semble n'avoir aucun rapport avec notre passage.

\subsection{La recréation du monde}

Après la courte parenthèse, organisée en Ringkomposition ${ }^{111}$ où le poète remonte dans l'histoire sacrée antérieure (démarche qui se justifie par l'intention du poète de souligner que Zeus intègre par engloutissement tout ce qui avait été créé avant lui), la narration se poursuit avec le récit des conséquences qui découlent de l'ingestion du phallus du Ciel. Dans le mythe hourrite, Koumarbi devenait enceint de cinq dieux après avoir dévoré le phallus du Ciel, Anou, et, dans notre poème, Zeus se retrouve enceint de toutes les potentialités génératives que contenait le phallus du premier dieu masculin, le Ciel. Mais les effets en sont ici beaucoup plus importants, car c'est bien toute la création qui se trouve bouclée dans le dieu :

108 Detienne - Vernant (1974), Cf. aussi Scalera McClintock (1988) 142, Casadesús (1996) $75 s q .$, Calame (1997) 73.

109 West (1983) 88; 114.

110 Cf. l'excellente argumentation de Betegh (1999) $\$ 1.5$.

$111 C f$. Betegh (1999) $₫ 1.6$. 


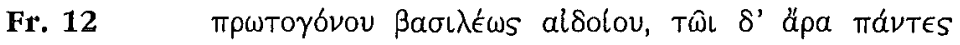

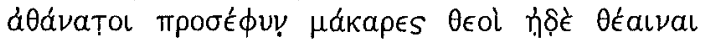

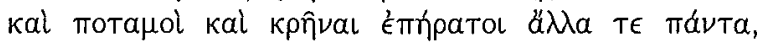

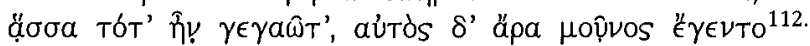

du phallus du roi aîné, et dans celui-ci tous les immortels se sont conçus ${ }^{113}$ : des dieux bienheureux et des déesses, des fleuves, des sources aimables et tout ce qui reste, tout ce qui était parvenu à être, c'est ainsi qu'il arriva à être le seul.

Il s'agit d'une espèce de grossesse cosmique. En avalant le phallus du Ciel, suivant les prophéties de la Nuit et de son père Kronos, Zeus remonte dans le temps, il remonte à l'origine et redémarre l'histoire de l'univers, en devenant la "nouvelle mère " de celui qui avait été l'aîné. Si le Ciel a bien été le premier né, Zeus, en quelque sorte, pour avoir introduit le phallus du Ciel dans son sein, devient lui-même l'ascendant de ce premier être, tout en ayant été le dernier-né. En effet, on dira dans le fr. 14, 1 «Zeus naquit le premier, Zeus, le dernier ».

Ayant avalé le phallus du Ciel, Zeus a la potentialité de l'univers entier, il tombe enceint de tous les êtres du cosmos. Il se peut que le poète ait prétendu avéc tout cela créer un modèle cyclique (étranger à Hésiode) d'alternance des temps ${ }^{114}$ et s'inscrire, de ce fait, dans une problématique très chère aux poètes cosmogoniques et aux présocratiques : celle de l'alternative de l'unité et la pluralité. Et ce modèle cyclique est peut-être en cohérence avec le message orphique, d'après lequel on doit traverser des périodes cycliques déterminées avant d'atteindre le salut définitif de l'âme. Mais tout ceci n'est qu'une simple possibilité. Encore que l'un et l'autre (le modèle cyclique du temps et de l'unité-pluralitét ${ }^{115}$ et le sort des âmes) coexistent dans l'œuvre d'Empédocle, un auteur qui présente une influence reconnue des modèles orphiques ${ }^{116}$. En tout cas, outre son rôle de démiurge, Zeus, pour avoir avalé le phallus du Ciel, conquiert le pouvoir, un pouvoir qu'aucun être ne va lui disputer car il est le dernier dieu, qui est devenu le premier

112 Col. XVI 3 sq. Dans le v. 4 đara est une lecture de Tsantsanoglou qui m’a été communiquée par lettre : [8]]бơa ZPE (1982).

113 Je préfère cette traduction de $\pi \rho \circ \sigma \epsilon \phi v \nu$ aux propositions de West, (1983) 88 (« became one with him, grew on to him », cf. Janko [2001] 25), de Scalera Mcclintock (1988) 148 (« divennero uno ») et de Casadesús (1995) 349s (« neixeren »).

114 Cf. Bernabé (1990).

115 Cf. Bernabé (1998b). Comme nous l'avons déjà vu, le commentateur remarque dans la col. XVI 8 sq. : «quant à l'expression 'c'est ainsi qu'il parvint à être l'unique chose au monde', il y met en évidence que le propre Intellect est la seule chose, parmi toutes les autres, à avoir vraiment de l'importance », en identifiant Zeus à l'Intellect et en essayant ainsi de relier la doctrine religieuse orphique aux théories d'un Anaxagore, par exemple.

116 Cf. West (1983) 108 qui suit les suggestions, par lettre, de Burkert. Au sujet des rapports d'Empédocle avec l'orphisme, cf. l'excellent travail de Riedweg (1995). 
grâce à sa capacité de s'auto-précéder, en altérant ainsi les normes de la succession au pouvoir ${ }^{117}$. Dans un autre fragment (qui suivait probablement le fragment 12 sans solution de continuité) ${ }^{118}$ la nouvelle situation est proclamée :

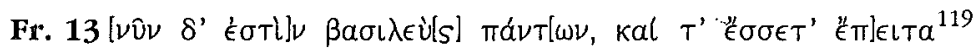

Il est maintenant le roi de tout et dorénavant il le sera.

Ce qui semble indiquer que, dans ce poème, le règne de Dionysos n'était pas abordé, au contraire des Rhapsodies.

\subsection{L' « Hymnè à Zeus"}

La référence à l'avénement du règne de Zeus sert à introduire un autre fragment, qui suivait fort probablement le fr. 13

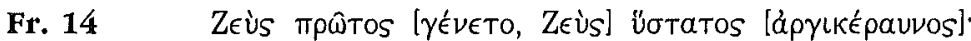

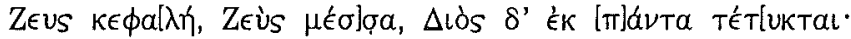

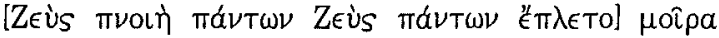

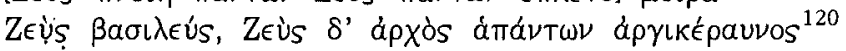

Zeus naquit le premier, Zeus, le dernier, à la foudre resplendissante

Zeus est la tête, Zeus le centre, Zeus dont tout s'est formé

Zeus, l'haleine de tout, Zeus de tout le destin,

Zeus souverain, Zeus le maître de tout, à la foudre resplendissante.

C'est un court hymne à Zeus inséré dans le poème. Avant la découverte du papyrus nous connaissions déjà plusieurs versions d'un hymne similaire, dont l'une est mentionnée par Platon ${ }^{121}$, elle était donc contemporaine ou 'légèrement postérieure à celle du Papyrus de Derveni, et une autre, plus

117 Cf. Bernabé (1989).

118 West (1983) $13 s q$., cf. aussi Burkert (1970) $445 s q$., Boyancé (1974) 109 sq., Funghi (1979) 24 sq., Alderink (1981) 28 sq., Calame (1997) 70.

119 Col. XVI 14. Les suppléments au texte sont dus à West.

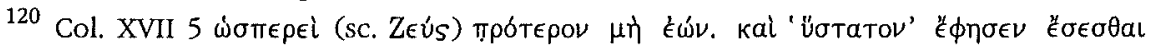

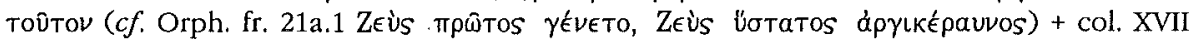

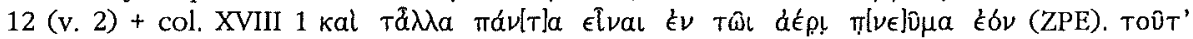

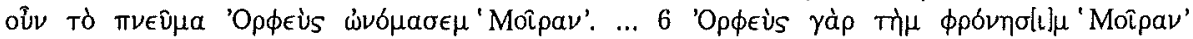

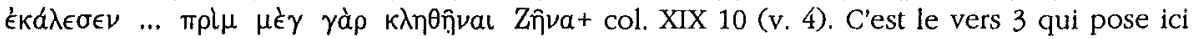
le plus de problèmes, il a été reconstitué par Merkelbach (1967) sur les bases du fr. 21a 5

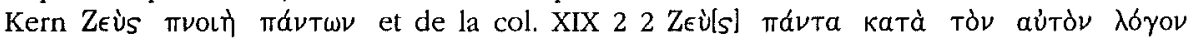

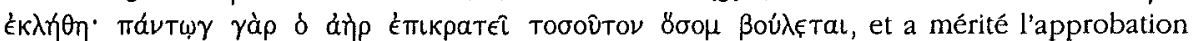
de Boyancé (1974) 97 sq. ( $\pi \nu \in \hat{0} \mu a$... « ne me paraît devoir être en prose autre chose que l'équivalent de ce que $\pi \nu$ ort est dans la langue poétique »), de Ricciardelli (1980) 118, de Funghi (1980) 80s et de Tsantsanoglou, per litteras. Toutefois West (1983) 90 n. 36, considère le v. 5 du fr. 21a comme une "Stoic interpolation ".

121 Plat. Leg. 4, 715e, bien que nous ne la connaissions toute entière que par le De mundo pseudo-aristotélicien et par Apul. De mundo 37. Cf. les fr. 21 et 21a Kern. 
tardive, avec un nombre de vers nettement supérieur, qui a été recueillie par des sources stoïciennes et néoplatoniciennes ${ }^{122}$. La trouvaille du papyrus vient nous montrer qu'une version, au moins, de l'hymne était déjà connue au $\mathrm{vI}^{\mathrm{e}}$ siècle av. J.-C. ${ }^{123}$ et qu'il faut, par conséquent, tempérer le penchant, répandu parmi les commentateurs modernes, à voir des « influences stoïciennes » dans les vers de l'hymne. Il existe probablement un rapport dialectique entre l'un et les autres. En effet, d'un côté, l'hymne a dû attirer l'attention des Stoïciens à cause des ressemblances de certains de ses contenus avec leurs propres théories, et, de l'autre, ces ressemblances auraient eu l'occasion de s'accroître, lors de versions postérieures, par des additions de type stoïcien.

Cet hymne est fondamentalement composé par des expressions polaires exprimant que Zeus est absolument tout, avec quelques résonances du langage d'Héraclite. Nous trouvons chez d'autres auteurs ${ }^{124}$ des parallèles à cette "religion de Zeus », plus évoluée que l'image d'Homère ne nous la transmet.

Il y est dit que Zeus fut le premier et le dernier à naître. Il est en effet, nous venons de le voir, le dernier rejeton de la chaîne de générations CielKronos-Zeus, mais, en avalant le phallus du Ciel et en devenant ensuite « enceint » de l'univers entier, il parvient à être le progéniteur de toute chose et, dans ce sens, le premier. Cette démarche de Zeus a renversé le cycle des générations. Aussi devient-il la tête et le centre de tout, comme s'il était une espèce d'air vivifiant toute chose ${ }^{125}$. En ce qui concerne l'identification de Zeus au destin ( $\mu$ oĩ $\rho$ ), elle est probablement due au fait que Zeus devient aussi le mâ̂tre de la destinée des choses ${ }^{126}$, en tant que leur démiurge, et encore de l'organisation du temps, ainsi que le signale Calame ${ }^{127}$. Les autres noms qu'on lui donne, son caractère de souverain, de seigneur de tout et de maître de la foudre resplendissante, sont absolument traditionnels.

D'autres vers venaient vraisemblablement poursuivre l'hymne à Zeus, dans le poème, qui servaient de transition au processus de création du

122 Fr. 168 Kern.

123 Mais ce qui n'est pas licite, c'est d'essayer de faire un hymne en réunissant toutes ses versions, comme l'a prétendu Forderer (1981).

124 Semon. fr. 1, 1 West, Iamblich. Protr. 4 (54, 19 Des Places = Ps.-Archytas De sapient. p. 45 Thesleff), Terpand. fr. 3 Gostoli (PMG 698, 1 Page), $c f$. Janko (1997) 83.

125 Dans l'analyse du passage effectuée par le commentateur, qui n'aide pas, à cette occasion, à améliorer notre connaissance du poème, l'inspiration philosophique principale est Diogène d'Apollonie et sa théorie de « l'air divin et intelligent».

126 Nous retrouvons une référence similaire dans celle qu'on a appelée la « Grande lamelle » de Thourioi, $c f$. l'interprétation que nous en avons récemment proposée dans Bernabé - Jiménez San Cristóbal (2002) 183 sq.

127 Calame (1997) 74. Alderink (1981) 28 à son tour, a signalé que Moira est un attribut propre à Zeus et, donc, non extérieur à lui-même, $c f$. aussi Ricchiardelli Apicella (1980) $118 \mathrm{sq.}$., Casadesús (1995) $381 \mathrm{sq}$. et surtout Betegh (1999) \$1.5; 2.2.2.2. 
monde mené à bien par le dieu ${ }^{128}$. West ${ }^{129}$ rapporte ici, exempli gratia, les derniers vers de l'hymne à Zeus de la version connue par l'auteur du De monde (fr. 21a 8-9 Kern).

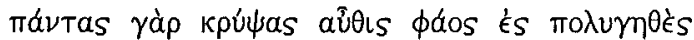

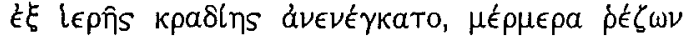

Car, après les avoir tous cachés, de son sein divin

il les rendit à nouveau à la très rejouissante lumière, auteur de merveilles.

Des vers très ressemblants à ceux-là, sinon les mêmes, devaient apparaître aussi dans notre poème.

\subsection{La naissance d'Aphrodite}

Pour que la création du monde puisse continuer à se produire, l'existence d'un principe générateur féminin devient nécessaire. Ceci expliquerait sans doute que Zeus, dans les premiers moments de sa démiurgie, ait généré la déesse Aphrodite comme responsable de ces fonctions. Nous ne disposons malheureusement pas des vers littéraux, mais bien, par contre, d'un passage en prose présentant de nombreuses allusions à ce moment, qui nous permet de deviner la suite de l'histoire :

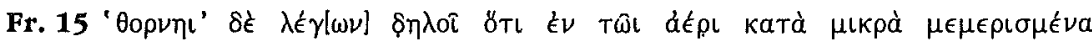

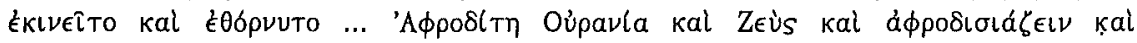

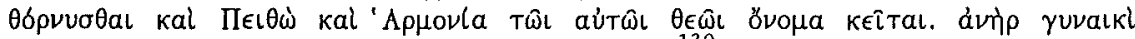

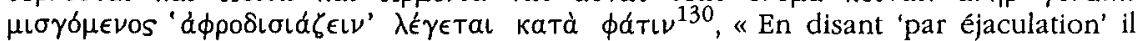
manifeste que dans l'air (les choses), une fois divisées en petites particules, bougeaient et 'sautaient'.... 'Aphrodite Ourania', Zeus, acte sexuel, éjaculer, 'Persuasion' et 'Harmonie' sont des noms qu'on a donnés au même dieu. Lorsqu'un homme s'unit à une femme on dit qu'il 'fait l'amour' en langage vulgaire ${ }^{131}$.

C'est donc ici Aphrodite, tout comme l'était Éros chez Hésiode, qui se porte garant, au début des temps, de la perpétuité de la race et, en général, de la fécondité sur la terre. Chez Hésiode, Aphrodite naît du membre mutilé du Ciel. Dans notre poème, il semble que sa naissance soit mise en rapport avec une certaine effusion de liquide séminal ${ }^{132}$, probablement de Zeus ${ }^{133}$. Il

128 Il s'agit de " a 'monistic' account on the origin of the world", selon Alderink (1981) 29s. Au sujet du dieu créateur chez les orphiques, $c f$. Guthrie (1952) 107 sq., Classen (1962) $9 s q .$, Alderink (1981) $25 s q .$, Parker (1995) 492.

129 West (1983) 115.

${ }^{130}$ Col. XXI 1 sq..

131 West (1983) 115 reconstitue également exempli gratia les vers auxquels le commentateur aurait fait allusion.

${ }^{132}$ La traduction de $\theta$ ó $\nu \nu \eta$ est conjecturale. Kapsomenos (1965) a interprété ce mot comme $\mu \in(\xi \in l$, Merkelbach (1967) 27 comme "Same ", Schwabl (1978) 1329 comme «Sprung, Besprung, Samensprung, Samen? », West (1983) 91 et n. 37 comme "from his seed " or «by an ejaculation », Laks - Most (1997) 19, n. 53 comme « by jumping/ mating ». Ce pourrait être aussi un verbe, comme le proposent, même avec des doutes, 
semble d'ailleurs logique que la naissance d'une divinité qui aura à s'occuper de la génération se rattache aux génitaux du dieu démiurge.

Il est fait également mention dans notre texte d'Harmonie et de Persuasion, qui, probablement, s'occupent d'Aphrodite au moment de sa naissance - comme Éros et Himéros (Amour et Charme) chez Hésiode (Théog. 201) - et qui représentent les aspects positifs et paisibles de la déesse de l'amour ${ }^{134}$.

\subsection{La recréation rationnelle de l'univers}

La recréation totale du monde devait se produire au moment où Zeus «accouchait» de tous les êtres, doué qu'il était d'une immense fécondité après avoir avalé le phallus du Ciel, ce qui explique «sa capacité de générer tous les éléments qui étaient nés pendant le règne de ses prédécesseurs ${ }^{135}$. Il a fait probablement renaître tout d'abord, comme dans les théogonies postérieures, le Ciel et la Terre, autour de laquelle Zeus installe l'Océan :

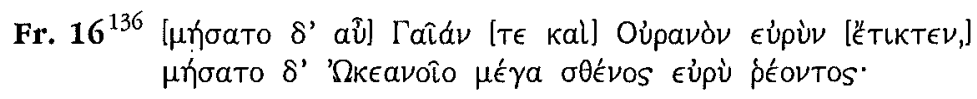

Calame (1997) 70 n. 7, Tsantsanoglou (1997) 10 n. 53 (il peut s'agir d'une forme erronée pour $\theta 6 \rho \eta$ ) ou Janko (1997) 64, (2001) 28 n. 164 (qui propose d'y lire $\theta 0 p v<u ́ r \eta \iota ~ « l e a p s »$ « mounted»). Cf. aussi Ricciardelli Apicella (1980) 122 sq., Casadesús (1995) 413 sq. au sujet toujours de cette forme difficile. West, lui, avait proposé (apud Merkelbach [1967] 27) qu'il s'agissait de la naissance d'Aphrodite, $c f$. Funghi (1979) $27 s q$., West (1983) $91 s q$.

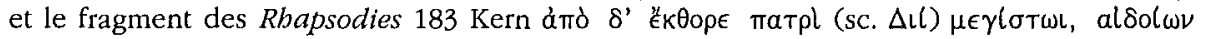

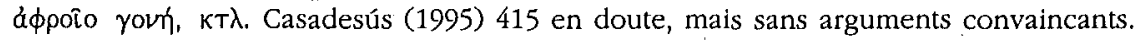

${ }^{133}$ Cf. West (1983) $91 s q$. Je ne partage pas les doutes de Betegh (1999) $₫ 2.2 .2 .3$, au sujet surtout de l'emploi de l'épithète Ourania. C'est une épithète traditionnelle qu'il faut mettre en rapport avec le fait que, si Zeus a avalé le phallus du Ciel, sa fécondité doit être celle du Ciel. Dans le poème hittite que j'ai cité plus haut, en le mettant en parallèle avec notre texte, Koumarbi avait craché une partie du liquide séminal contenu dans le membre dévoré d'Anou (le Ciel), ce qui fit naître un dieu. $C f$. Bernabé (1987) $148 \mathrm{sq}$.

${ }^{134}$ Betegh (1999) $₫$ 2.2.2.5 tente de placer Harmonie et Persuasion dans la généalogie.

135 Comme le signale Calame (1997) 72.

${ }^{136}$ Le premier vers est rétabli ici par West (1983) 115, Tel que Betegh (1999) $₫ 2.2 .2 .2$, le signale, le papyrus atteste que la Terre était mentionnée dans la col. XXII $7 \Gamma \tilde{\eta} \delta \xi \mathrm{kal}$

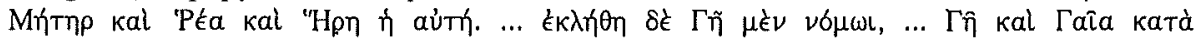

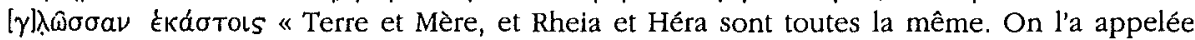
Terre par convention, ..... Gè et Gaia, selon le dialecte de chacun ". Quant au Ciel, $c f . c o l$.

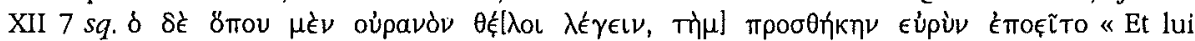
(Orphée), lorsqu'il veut dire 'ciel', emploie l'épithète 'vaste' ». Le vers 2 fut rétabli par

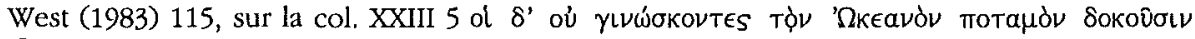

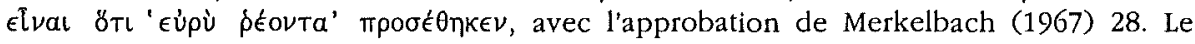
papyrus cite ensuite le vers 4, introduit par Tò $\delta^{\prime}$ ' $\chi \chi \delta \mu \epsilon \nu_{0} \nu$ « le suivant ». Quant au début du vers 5, il ne provient pas du Papyrus de Derveni, mais de P.Oxy. 221, 9, 1, qui contient un commentaire de l'nlliade d'Homère. Tsantsanoglou m'avait communiqué (par lettre) son observation que le commentateur homérique y citait le vers 3 de notre texte, suivi des mots qui nous ont permis de reconstruire le début du vers 4. 


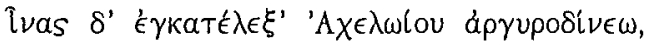

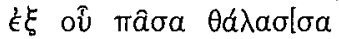

Il conçut aussi la terre et engendra le vaste Ciel.

et conçut la puissante force d'Océan au large courant

et fit couler dans celui-là les nerfs d'Achéloos aux remous argentins,

dont toutes les mers proviennent...

Mais ce qui est plus intéressant est que cette nouvelle création nous montre un caractère rationnel et ordonné. En effet, Zeus nous y est présenté comme un démiurge qui obéit, cette fois-ci, à un dessein préconçu et intelligent $^{137}$, face à la situation antérieure que l'on suppose plus « chaotique ", plongée dans la violence et le désordre. Pour renforcer cette idée, le poète utilise le très intéressant verbe $\mu$ ñбato que j'ai traduit par « il conçut », dans le sens de « concevoir intellectuellement ${ }^{138}$, en m'appuyant sur le double sens, intellectuel et sexuel, du verbe. Il s'agit moins ici d'une nouvelle création « intellectuelle ${ }^{139}$, que de $«$ a mental act of planning and contriving ${ }^{140}$. N'oublions pas que Zeus, après son action dévoratrice, réunit en lui-même non seulement le pouvoir des dieux mais aussi leur $\mu$ ๆ̂tıs (fr. 11). Il existe ici un clair rapport étymologique que le poète met en relief.

En plus d'Océan ${ }^{141}$ naissent « les nerfs de l'Achéloos », une belle métaphore du réseau de fleuves qui sillonne la terre ${ }^{142}$. Achéloos est fréquemment employé pour « eau » ou bien « eau primordiale universelle ${ }^{143}$ dont naissent toutes les mers ${ }^{144}$.

137 Cf. Tarán (1971) 407 n. 162

138 Nous retrouvons des emplois similaires de ce même verbe ou d'autres du même

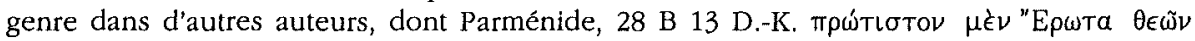

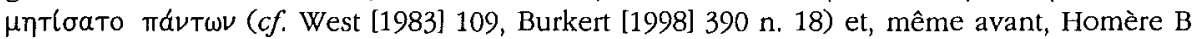

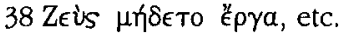

139 Scalera McClintock (1988) 143.

140 Tarán (1971) 407 n. 162 ( $c f$. sa note du fr. 13 de Parménide), $c f$. aussi Burkert (1968) 102 n. 16, (1969) 3 n. 7, (1997) 173, Schwabl (1978) 1330 (qui signale que, chez les orphiques se produit la coïncidence de la pensée et de son éxécution), Ricciardelli Apicella (1980) 125 sq. et n. 82, Casadesús (1995) 453.

${ }^{141}$ Betegh (1999) 2.2.2.4 pense concevable qu'il soit fils d'Océan, comme le sont tous les fleuves chez Hésiode, mais il n'y a pas de traces de cette question dans notre texte.

142 Cf. West (1983) 92.

143 Cf. Serv. in Georg. 1, 8; Eur., Andr., 167; Bacch. 625; Hypsip. p. 73 Cockle; Panyas., fr. -31 Bernabé; Schol. Hom. $\$ 194$ (V 166 Erbse), Schol. Genov. Hom. $\Phi 195$ (I 198, 15 Nicole); Schol. Hom. $\Omega 616$ (V 624 Erbse); Eust. in Il. p. 1231, 12, etc.

${ }^{144}$ C'est probablement à un passage comme celui-ci que font référence toutes les discussions des théologiens sur les sources de la mer qui apparaissent chez Aristote et chez Alexandre d'Aphrodise (Aristot., Meteor., 353a 34; Alex. Aphrod, in Aristot. Meteor. 66, 12; 67, 23; 70, 35 Hauduck; Quaest. 98, 20 Bruns). 
La lune est une autre divinité-être physique qui naît de Zeus. Dans les Rhapsodies (fr. $91 \mathrm{Kern}$ ) nous retrouvons des vers similaires où il est dit que Zeus « conçut la lune »:

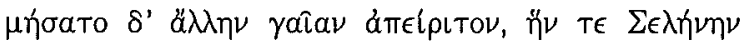

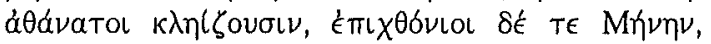

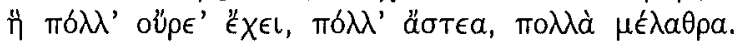
Il conçut aussi une autre terre immense, appelée Lune par les immortels, et Mène par les habitants de la terre ${ }^{145}$, possédant beaucoup de montagnes, beaucoup de villes et de toits.

Il n'est pas impensable que le poème cité dans le Papyrus de Derveni ait déjà contenu ces mêmes vers. Un passage attribué par les doxographes à Héraclide du Pont, le disciple d'Aristote ${ }^{146}$, semble venir à l'appui de l'ancienneté de cette curieuse conception lunaire, digne d'un Lucien ou d'un Cyrano de Bergerac, s'il est toutefois vrai que kó $\sigma$ ov traduit l'idée que les astres sont habités et si l'on considère encore que la Lune en fait partie :

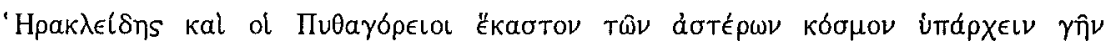

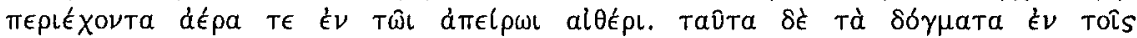

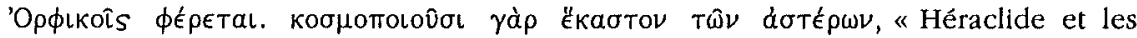
Pythagoriciens affirment que chaque astre constitue un monde : et que la terre est entourée d'air dans l'éther illimité. De telles opinions se retrouvent dans les poèmes orphiques, car on y fait de chaque astre un monde $»$.
}

Mais ne spéculons pas. Le commentateur ne nous permet de reconstituer que peu de choses autour de la référence à la Lune dans ce poème :

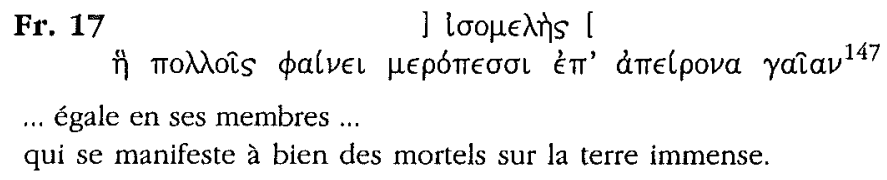

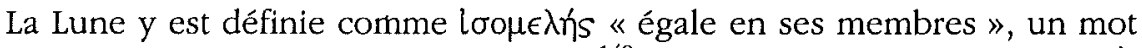

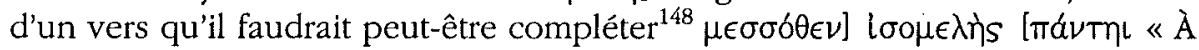

145 Nous y voyons une référence au fait qu'll existe une langue des dieux différente de celle des mortels, $c f$. à cet égard Bernabé (1992b) 31 sq., avec bibliographie. On attribue ici aux hommes le nom habituel de la lune ( $\Sigma \in \lambda \dagger \eta \eta \eta$, c'est-à-dire «celle qui resplendit») et aux dieux celui de Mrim (qui se rapporte au mot désignant en grec le « mois», car la lune y est considérée comme l'unité de mesure d'un mois, en tant que cycle lunaire).

146 Aët., Plac. 2, 13, 15 = Plut., de plac. philos., 2, 13; Stob., Flor., 1, 24, 1 (I 204, 21 Wachsm. = Doxogr. 343,7 = Heraclid., fr. 113a Wehrli), cf. aussi Galen., Histor. philos., 56 (Doxogr. 624, 15 = fr. 113c Wehrli).

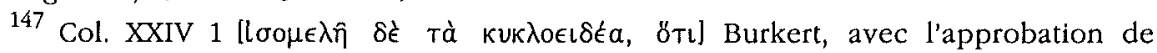

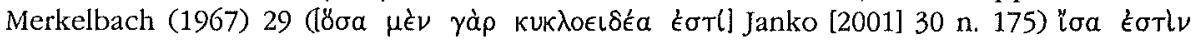

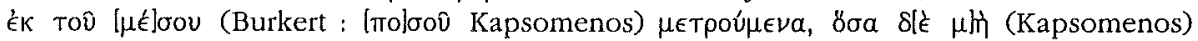

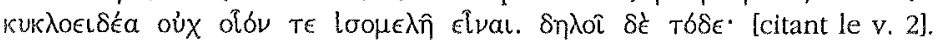

143 C. West (1983) 115. 
partir de son centre égale en ses membres de tous côtés » ou de forme similaire, dans le sens où elle est sphérique. Il y est dit aussi que « beaucoup de gens » la voient. Si l'on n'y parle pas de «tous » les êtres de la terre c'est vraisemblablement parce qu'elle ne peut être vue de tout le monde en même temps $^{149}$. En outre, la lune est étroitement associée à la mesure du temps, dès lors qu'elle en donne l'une des unités de base : le mois. C'est ainsi que la création de Zeus met également en place le cadre chronologique de l'univers.

\subsection{L'inceste}

Deux autres vers marquent un nouveau tournant de l'histoire :

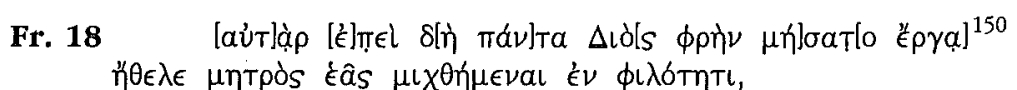

Mais une fois que l'esprit de Zeus eut conçu toute son œuvre

il voulait s'unir amoureusement à sa propre mère.

Avec son habituelle "vitesse narrative " l'auteur, qui vient tout juste de terminer la création de Zeus (racontée, elle aussi, en très peu de vers), aborde, sans solution de continuité, le thème de l'inceste du dieu avec sa mère. Il y fait à nouveau usage du verbe $\mu$ ñoato qui nous montre le caractère « intellectuel » et ordonné de la recréation du monde menée à bien par le dieu.

Aucun nom n'est donné dans le poème à la mère du dieu. Mais selon la tradition commune c'est Rhéa, comme chez les orphiques, encore que dans les Rhapsodies elle soit identifiée à Déméter ${ }^{151}$. Dans le commentaire elle est identifiée aussi avec Déméter et avec la Terre Mère ${ }^{152}$. Nous ignorons si cela répond à quelque chose d'explicite dans le poème commenté ou si c'est le seul fruit du travail d'analyse du commentateur.

149 Cf. West (1983) 93, «I wonder whether he imagined that the moon's phases were different as seen from different parts of the earth, so that there were always some peoples to whom it was invisible. "

${ }^{150} \mathrm{La}$ reconstitution de ce vers est due à Tsantsanoglou, qui me l'a communiquée par lettre et est réalisée à partir de quelques lettres qu'on arrivait à lire dans la col. XXV 14. Celle du vers suivant appartient à West (1983) 115 , effectuée sur la base de la col. XXVI 1

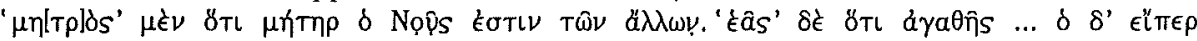

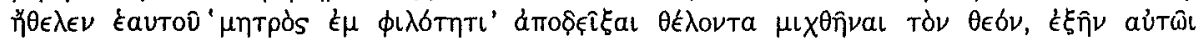

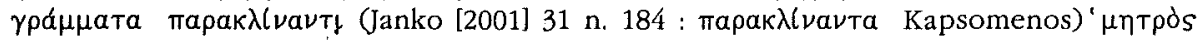

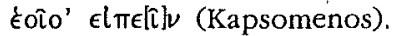

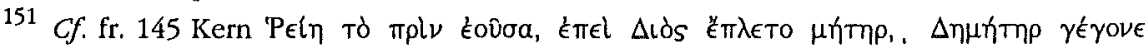
« celle qui avait été Rheia est devenue Déméter ».

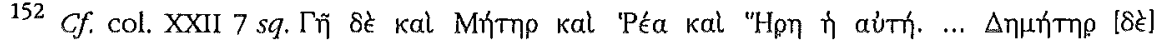

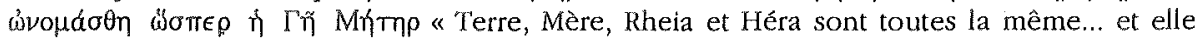
sappelle Déméter, tout comme Ge Meter. » 
Nous ignorons également le but de cette union incestueuse ${ }^{153}$. Malheureusement notre information sur le poème orphique s'interrompt ici et il ne nous reste donc que la possibilité de spéculer sur les motivations de Zeus. Il semble possible qu'il ait voulu s'unir à sa mère pour revenir en arrière sur le plan sexuel aussi; en effet, en épousant sa propre mère, il devient une espèce de père pour lui-même. Plusieurs raisons pourraient justifier cette attitude, dont l'une pourrait être la simple prévoyance face à ses héritiers, car, en devenant l'époux de sa mère il rompt un cycle de successions où les rois précédents avaient perdu la souveraineté, chacun à son tour, sous les coups de son propre fils ${ }^{154}$. Une autre explication pourrait être le fait qu'il annulait ainsi la distinction entre les deux moments de la création du monde ${ }^{155}$. Et une autre encore serait sa volonté d'engendrer Dionysos et qu'en engendrant d'abord Perséphone qui lui naît de sa propre mère et puis Dionysos, issu de Perséphone (laquelle serait donc sa fille et son épouse en même temps), il réussit en quelque sorte à faire que Dionysos appartienne à la même génération que lui (c'est-à-dire qu'ils auraient le même rang). Mais nous avons bien vu qu'un règne de Dionysos n'est pas cohérent avec ce que nous lisons dans le texte. Ceci nous amène directement au problème des suites possibles de l'histoire.

\subsection{Aurait-elle une suite, cette histoire?}

Il nous reste à poser ici la question de l'hypothétique suite du poème. Nous ne pouvons ni affirmer ni nier la possibilité que d'autres sujets propres à la tradition orphique postérieure n'aient été abordés ici, dont la naissance de Perséphone ou celui d'une nouvelle union incestueuse de Zeus avec cette déesse afin d'engendrer Dionysos. Dans les Rhapsodies, on met même en rapport l'histoire théogonique avec des thèmes relatifs au salut des âmes, mais il est trop risqué d'extrapoler, sans une critique préalable, dans un' poème du $\mathrm{IV}^{\mathrm{e}}$ siècle av. J.-C., des traits propres à un autre poème qui lui est postérieur de quatre siècles.

D'un autre côté, cette question n'est pas sans rappoít avec l'existence possible d'un deuxième rouleau contenant la suite du commentaire (et, donc, des citations du poème) qui aurait brûlé sur le bûcher funéraire ${ }^{156}$. Mais ce deuxième rouleau n'est qu'une entéléchie, aussi est-il préférable de ne pas compter sur un élément dont l'existence n'a même pas été constatée. Je

153 Le commentaire ne nous vient pas ici en aide, car le commentateur, dans le but d'atténuer la brutalité de l'incestẹ, a prétendu "retraduire » le passage en suivant une fausse étymologie (d'après laquelle éâs ne signifierait pas "sa ", comme le pensent, assure-t-il, "ceux qui ne comprennent pas l'expression », mais « bonne »).

154 Au sujet des rapports qui se produisent entre la lutte pour le pouvoir divin et les essais d'altérer le cours normal des générations, $c f$. Bernabé (1989).

155 Calame (1997) 74.

156 Cf. Janko (2001) 32 n. 190. 
pense, d'ailleurs, qu'il est très probable, vu la vitesse narrative du poète, qu'il se soit limité, dans son texte, à indiquer les faits, tout en confiant la reconstruction de la totalité de l'histoire à la " compétence » intertextuelle de son auditoire.

\section{7. Évaluation}

La théogonie commentée dans le Papyrus de Derveni est, nous l'avons vu, un texte fascinant qui, malheureusement, nous est parvenu sous une forme particulièrement fragmentée et incomplète, dont la reconstitution nous oblige encore à en préciser quelques détails, en partant toujours de ce que notre commentateur anonyme nous permet d'entrevoir à son sujet dans son analyse. Cette théogonie constitue un mythe complexe et très attrayant sur l'organisation du monde et du pouvoir divin au début des temps, qui présente de nombreuses coïncidences avec la Théogonte d'Hésiode - le poème représentatif de ce type de littérature le mieux connu -, mais aussi de notables divergences avec celui-ci. Du point de vue religieux, la grande nouveauté de cet ouvrage consiste à nous présenter un Zeus qui n'est pas seulement la divinité suprême de l'univers, mais aussi son démiurge, ayant obéi, lors de sa création, à un dessein préétabli et intelligent, tout pittoresques qu'aient été les moyens dont il s'est servi pour y parvenir; à savoir, avaler le phallus de celui qui avait été le premier dieu, pour pouvoir ainsi remonter dans le temps juste avant l'origine de tout et recommencer la création du monde..

Du point de vue littéraire, c'est un beau texte, où l'on découvre des échos traditionnels mais où d'autres apparaissent, qui nous rappellent la poésie philosophique et qui nous permettent, tout au moins, d'entrevoir le type de sources où durent puiser Parménide et Empédocle, pour ne citer ici que les cas les plus notables. Son caractère profond et allusif nous amène à supposer l'existence d'un ensemble plus vaste d'ouvrages, comprenant des textes montrant tous la même tendance, dont aucun n'aurait une longue étendue, mais qui seraient complémentaires les uns des autres, tissant, tous ensemble, un riche réseau d'intertextualités. Tout un ensemble littéraire dont nous ne pouvons que déplorer la perte et l'oubli, dans le tumulte qui caractérise la transmission des textes de l'antiquité ${ }^{157}$.

Alberto BERNABÉ

Universidad Complutense

Departamento di Filología Griega y Lingüistica Indoeuropea

Ciudad Universitaria

E - 28040 Madrid

albernab@filol.ucm.es

157 A propos de la perte des ouvrages orphiques, cf. Bernabé (1993). 


\section{Références bibliographiques}

L.J. Alderink, Creation and salvation in ancient Orphism, Chico, 1981.

R. Baumgarten, Heiliges Wort und Helige Schrift bei den Griechen, Tübingen, 1998.

T.M.S. BAXTER, The Cratylus. Plato's critique of naming, Leiden/New York/Köln, 1992, p. 130-139.

A. Bernabé, Textos literarios hetitas, Madrid, $1987^{2}$.

-, "Generaciones de dioses y sucesión interrumpida. El mito hitita de Kumarbi, la “Teogonía' de Hesíodo y la del 'Papiro de Derveni'", $\operatorname{AOr} 7$ (1989), p. 159-179.

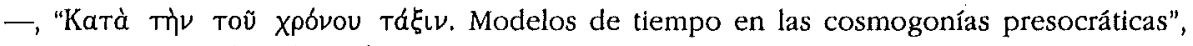
Emerita 58 (1990), p. 61-98.

-, "La poesía órfica: un capítulo reencontrado de la literatura griega", Tempus 0 (1992), p. 5-41 (1992a).

-, "Una forma embrionaria de reflexión sobre el lenguaje: la etimología de nombres divinos en los órficos", RSEL 22 (1992), p. 25-54 (1992b).

-, "Consideraciones sobre la épica griega perdida", in J.A. López FÉrez (éd.), La épica griega y su influencia en la literatura española (Aspectos literarios, sociales y educativos), Madrid, 1993, p. 155-188.

—, "Una etimología platónica: $\omega \hat{\omega} \mu \alpha-\sigma \hat{\mu} \mu "$, Pbilologus 139 (1995), p. 204-237.

-, "Plutarco e l'orfismo", in I. Gallo (éd.), Plutarco e la Religione, Atti dell VI Convegno plutarcheo (Ravello, 29-31 maggio 1995), Napoli, 1996, p. 63-104 (1996a).

-, "La fórmula órfica 'cerrad las puertas, profanos'. Del profano religioso al profano en la materia",' Ilu. Revista de ciencias de las religiones 1 (1996), p. 13-37 (1996b).

-, "Platone e l'orfismo", in G. Sfameni Gasparro (éd.), Destino e salvezza: tra culti pagani e gnosi cristiana. Itinerari storico-religiosi sulle orme di Ugo Bianchi, Cosenza, 1997, p. 37-97 (1997a).

-, "Orfeotelestas, charlatanes, intérpretes: transmisores de la palabra órfica", Homenatge a Miquel Dols, Palma de Mallorca, 1997, p. $37-41$ (1997b).

-, "Las Noches en las Rapsodias órficas", Actas del IX Congreso de la Sociedad Española de Estudios Clásicos. Madrid V (1998), p. 71-76 (1998a).

-, "Lo uno y lo múltiple en la especulación presocrática: nociones, modelos y relaciones", Taula, quaderns de pensament (UIB) 27-28 (1998), p. 75-99 (1998b).

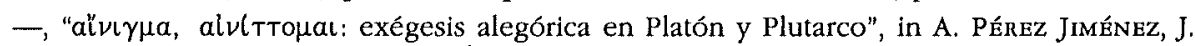
García lópez, R. Agullar, Plutarco, Platón y Aristóteles, Actas del V Congreso Internacional de la International Plutarch Society (Madrid-Cuenca, 4-7 de mayo de 1999), Madrid, 1999, p. 189-200 (1999a).

-, "Una cita de Píndaro en Platón Men. 81 b (Fr. 133 Sn.-M.)", in J.A. López Férez (éd.), Desde los poemas boméricos hasta la prosa griega del siglo IV d.C. Veintiséis estudios filológicos, Madrid, 1999, p. 239-259 (1999b).

-, "La experiencia iniciática en Plutarco", in A. Pérez Jiménez, F. Casadesús (éds), Estudios sobre Plutarco: Misticismo y religiones mistéricas en la obra de Plutarco (Actas del VII Simposio Español sobre Plutarco), Madrid-Málaga, 2001, p. 5-22 (2001a).

-, "El papiro de Derveni", in De Tales a Demócrito. Fragmentos presocráticos, Madrid $2001^{2}$ (2001b).

A. Bernabé, A.I. Jiménez San Cristóbal, Instrucciones para el más allâ: Las laminillas órficas de oro, Madrid, 2002. 
G. Betegh, Cosmology, theology, and exegesis in the Derveni Papyrus, Ph. D. Thesis, Paris/Budapest, 1999.

J. BINGEN, in CE 42 (1967), p. 214.

W.E. Blake, "Oldest Greek Papyrus Discovered? ", CW 55 (1961-1962), p. 161.

R. BöHME, "Homer oder Orpheus?", ZPE 71 (1988), p. 25-31.

-, "Neue Orpheusverse auf dem Derveni-Papyrus", Emerita 57 (1989), p. 211-238.

A. Botrini, Arcbeologia della salvezza. L'escatologia greca nelle testimonianze archeologiche, Milano, 1992, p. 135-148.

P. Boyancé, "Remarques sur le papyrus de Derveni", REG 82 (1974), p. 91-110.

L. Brisson, "Les théogonies orphiques et le papyrus de Derveni. Notes critiques", RHR 202 (1985), p. 389-420 (= Orphée et l'Orphisme dans l'Antiquité gréco-romaine, Aldershot, 1995, I).

—, Orphée. poèmes magiques et cosmologiques, postface de -, Paris, 1993.

W. Burkert, "Orpheus und die Vorsokratiker. Bemerkungen zum Derveni-Papyrus und zur pythagoreischen Zahlenlehre", AEA 14 (1968), p. 93-114.

-, "Das Proömium des Parmenides und die Katabasis des Pythagoras", Pbronesis 14 (1969), p. 1-30.

-, "La genèse des choses et des mots. Le papyrus de Derveni entre Anaxagore et Cratyle", EPb 25 (1970), p. 443-455.

-, "Neue Funde zur Orphik", Information zum Alspracblicbe Unterricht 2, Graz, 1980, p. 27-41.

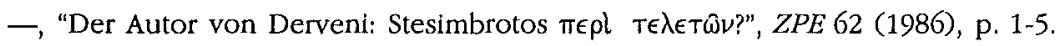

-, "Oriental and Greek Mythology: The meeting of parallels", in J. Bremmer (éd.), Interpretations of Greek Mythology, London-Sydney, 1987, p. 10-40.

-, "Star Wars or One Stable World? A Problem of Presocratic Cosmogony (PDerv. Col. XXV)", in Laks - Most (1997), p. 167-174.

-, "Die neuen orphischen Texte: Fragmente, Varianten, 'Sitz in Leben'", in W. BurkerT, L. Gemelli Marciano, E. Matelli, L. Orelli (éds), Fragmentsammlungen philosopbischer Texte der Antike, Göttingen, 1998, p. 387-400.

- Da Omero ai Magi. La tradizione orientale nella cultura greca, Venezia, 1999.

C. Calame, "Eros initiatique et la cosmogonie orphique", in Ph. Borgeaud (éd.), Orpbisme et Orpbée, en l'bonneur de Jean Rudbardt, Genève, 1991, p. 227-247.

-, "Figures of Sexuality and Initiatory Transition in the Derveni Theogony and its Commentary", in Laks - Most (1997), p. 65-80.

F. CASADESús, Revisió de les principals fonts per a l'estudi de l'orfisme a l'epoca classica (Plató $i$ el Papir de Derveni), Tesi Doctoral, Universitat Autònoma de Barcelona, Bellaterra, 1995.

-, "Metis, el nous, el aire y Zeus en el papiro de Derveni", Faventia 18 (1996), p. 75-88.

- "Nueva interpretación del Crátilo platónico a partir de las aportaciones del Papiro de Derveni", Emerita 68 (2000), p. 53-71.

G. Casadio, "Adversaria Orphica. A proposito di un libro recente sull'Orfismo", Orpbeus 8 (1987), p. 381-395.

-, Storia del culto di Dioniso in Angolide, Roma, 1994.

C.J. Classen, "The creator in Greek thought from Homer to Plato", Class. E Med. 23 (1962), p. 1-22.

S. Colabella, "Sul papiro di Derveni", in A. Masaracchia (éd.), Orfeo e l'orfismo, Roma, 1993, p. $67-75$ 
E. CondurachI, in Orfismo in Magna Grecia, Atti del XIV Convegno di Studi sulla Magna Grecia, Taranto 6-10 ott. 1974, Napoli, 1975, p. 184 sq.

G. DAux, "Chronique des fouilles 1961, Macédonie occidentale et centrale", BCH 86 (1962), p. 792 sq.

M. Detienne, J.-P. Vernant, Les ruses de l'intelligence. La mètis des Grecs, Paris, 1974.

F. Dunand, Le culte d'Isis dans le bassin oriental de la Méditerranée, Leiden, 1973.

M.J. Edwards, "Notes on the Derveni commentator", ZPE 86 (1991), p. 203-211.

C. Eggers Lan, "Los estudios sobre el orfismo clásico", Métbexis 4 (1991), p. 101-113.

M. Forderer, "Der orphische Zeushymnus", in G. KuRz (éd.), Gnomosyne, Festschrift für W. Marg, München, 1981, p. 227-234.

M.S. Funghi, "Una cosmogonia orfica nel Papiro di Derveni", PP 34 (1979), p. 17-30.

-, "Phronesis nel Papiro di Derveni", Papyrologica Florentina VII, Miscellanea Papyrologica, Firenze, 1980, p. 79-86.

-, "Esegesi di testi orfici", Corpus dei Papiri Filosofici Greci e Latini III, Firenze, 1995, p. 565-585.

—, "The Derveni Papyrus", in Laks - Most (1997a), p. 25-37.

—, "Bibliography of the Derveni Papyrus", in LaKs - Most (1997a), p. 175-185.

D. Gambarara, "Réflexion religieuse et réflexion linguistique aux origines de la philosophie du langage", in S. Auroux, M. Glatigny; A. Joly, A. Nicolas, I. Rosier, Matériaux pour une bistoire des théories linguistiques, Lille, 1984, p. 105-114 (1984a).

-, Alle fonti della filosofia del linguaggio, Roma, 1984 (1984b).

F. Graf, compte rendu de West (1983), Gnomon 57 (1985), p. 586-591

W.K.C. GuTHrie, Onpheus and Greek Religion, London, 1935 (1952 $2^{2}=$ New York, 1967).

A. Henrichs, "The Eumenides and wineless libations in the Derveni papyrus", Atti del XVII Congresso Internazionale di papirologia, Napoli, 1984, II, p. 255-268.

M. Henry, "The Derveni commentator as literary critic", TAPhA 116 (1986), p. 149-164.

M.S.F. Hood, "Archaeology in Greece 1961-1962", JHS, Archaeol. Reports for 1961-1962, p. 15.

H. Hunger, "Papyrusfund in Griechenland", CE 37 (1962), p. 415 sq.

E. Hussey, "The enigmas of Derveni", Oxford studies in ancient philosophy 17 (1999), p. 303-324.

R. JANKo, compte rendu de WEST (1983), CPh 81 (1986), p. 154-159.

-, "The Physicist as Hierophant: Aristophanes, Socrates and the Authorship of the Derveni Papyrus", ZPE 118 (1997), p. 61-94.

-, "The Derveni Papyrus (Diagoras of Melos, Apopyrgizontes logol?): A new translation", CPh 96 (2001), p. 1-32.

Ch. KaHN, "Language and ontology in the Cratylus", in E.N. LeE, A.P.D. Mourelatos, R.M. RorTy (éds), Exegesis and Argument. Studies Vlastos, Assen, 1973, p. 156-158.

-, "Les mots et les formes dans le 'Cratyle' de Platon", in Philosophie du langage et grammaire dans l'antiquité, Grenoble/Bruxelles, 1986, p. 91-103.

-, "Was Euthyphro the Author of the Derveni Papyrus?", in Laks - Most (éds) (1997), p. 55-63.

S.G. Kapsomenos, "Der Papyrus von Dervéni. Ein Kommentar zur Orphischen Theogonie", Gnomon 35 (1963), p. 222 sq.

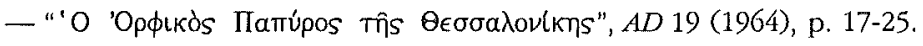

-, "The Orphic papyrus roll of Thessalonica", BASP 2 (1964-1965), p. 3-12. 
G.S. Kirk, J.E. Raven, M. Schofield, The Presocratic Philosophers, Cambridge, $1983^{2}$, p. 3033.

A. LAKS, "Between religion and philosophy: the function of allegory in the Derveni papyrus", Phronesis 42 (1997), p. 121-142.

A. Laks, G.W. Most (éds), Studies on the Derveni papyrus, Oxford, 1997.

A. Lamedica, "Il Papiro di Derveni come commentario. Problemi formali", in A.H.S. ElMosalamy (ed.), Proceedings of the XIXth International Congress of Papyrology, Cairo, 1989 [1992], I, p. 325-333.

-, "La terminologia critico-letteraria dal Papiro di Derveni ai Corpora scoliografici", in P. Radici Colace, M. Caccamo Caltabiano, Atti del I seminario di studi sui lessici tecnici greci e latini, Messina, 1991, p. 83-91

E. LAROCHE, Textes mythologiques bittites en transcription, Paris, 1965.

P. LÉvÊQue, "Religion poliade et sectes", Kemos 10 (1997), p. 233-240.

A.A. Long, "Stoic readings of Homer", in R. Lamberton, J.J. Kenney, Homer's ancient readers, Princeton, 1992, p. 41-66.

C.I. Makaronas, nota arqueológica in $A D 18$ (B) (1963), p. 193 sq.

B.G. Mandilaras, Пamúpol kal Пamupoגoyia, Athina, $1994^{2}$.

R. Merkelbach, "Der orphische Papyrus von Derveni", ZPE 1 (1967), p. 21-32.

G.W. Most, "The fire next time. Cosmology, allegoresis, and salvation in the Derveni Papyrus", JHS 117 (1997), p. 117-135.

D. Obbink, "A quotation of the Derveni papyrus in Philodemus' On Piety", CronErc 24 (1994), p. 111-135.

-, "Cosmology as Initiation vs, the Critique of orphic Mysteries", in Laks - Most (1997), p. 49-54.

E.N. Oschenschlager, "The Dherveni Finds", CW 56 (1963), p. 245-247.

R. PArker, "Early Orphism", in A. Powell (éd.), The Greek World, London, 1995, p. 483510.

R. PFEIFFER, History of Classical Scholarship, From the Beginnings to the End of the Hellenistic Age, Oxford, 1968.

D.M. PIPPIDI, "în jurul papirilor de la Derveni şi Callatis", StudClas 9 (1967), p. 203-210.

F. Prontera, "Sulla laminetta di Hipponion", PP 33 (1978), p. 48-58.

C. Ramnoux, La Nuit et les enfants de la Nuit, Paris, $1986^{2}$.

G. Ricciardelli Apicella, "Orfismo e interpretazione allegorica", Bollclass $3^{a}$ ser. 1 (1980), p. 116-130.

-, "Le teogonie orfiche nell'ambito delle teogonie greche", in A. Masaracchia (éd,), Orfeo e l'orfismo, Roma, 1993, p. 27-51,

N.J. Richardson, "Homeric Professors in the age of the Sophists", PCPhS 201 (1975), p. 6581.

Ch. RIEDWEG, Jüdisch-hellenistische Imitation eines orphischen Hieros Logos, Tübingen, 1993.

—, "Orphisches bei Empedokles", $A \mathcal{E} A 41$ (1995), p. 34-59.

J.S. Rusten, "Phanes-Eros in the Theogony of 'Orpheus' (PDerveni col. IX 4)", Alti del XVII Congresso Internazionale di Papirologia, Napoli, 1984, p. 333-335.

—, "Interim notes on the papyrus from Derveni", HSCPh 89 (1985), p. 121-140.

G. Scalera McClintock, "La teogonia di Protogono nel Papiro Derveni. Una interpretazione dell'orfismo", Filosofia e Teologia 1 (1988), p. 139-149. 
M. Schmid, intervention au colloque Orfismo in Magna Grecia, Atti del XIV Convegno di Studi sulla Magna Grecia, Taranto 6-10 ott. 1974, Napoli, 1975, p. 230.

H. SCHWABL, s.v. Zeus, RE Suppl. XV (1978), p. 1326-1330.

D. Sider, "Heraclitus in the Derveni Papyrus", in Laks - Most (1997), p. 129-148.

R. SOREL, Orphée et l'orphisme, Paris, 1995.

W. SPeyer, Bücherfunde in der Glaubenswerbung der Antike, Göttingen, 1970.

M. GHidini Tortorelli, "Eufrone: una divinità orfica? ", $P P 40$ (1985), p. 419-425.

-, "Nephele: una metafora orfica arcaica", $P P 44$ (1989), p. 29-36

-, "Due nuovi teonimi orfici nel papiro di Derveni", in Ph. Borgenud (éd.), Orphisme et Orphée en l'bonneur de Jean Rudhardt, Genève, 1991, p. 249-261.

L. TarÁn, "The Creation Myth in Plato's Timaeus", in J.P. Anton, G.L. Kustas, Essays in Ancient Greek Philosopby, Albany, 1971, p. 372-407.

K. Tsantsanoglou, "The First Columns of the Derveni Papyrus and their Religious Significance", in LaKs - MOST (1997), p. 93-128.

K. Tsantsanoglou, G.M. Parássoglou, "Heraclitus in the Derveni Papyrus", in Aristoxenica, Menandrea, Fragmenta Philosophica, Firenze, 1988, p. 125-133.

- -, "Heraclitus 1T", in Corpus dei Papiri filosofici greci e latini, I 1, Firenze, 1992, p. 221-226.

R. TurCan, s.v. Phanes, LIMC VII 1 (1994), p. 363 sq.

E.G. TURner, Greek manuscripts of the ancient world, Oxford, 1971.

- , "Ptolemaic bookhands", SGC 4 (1980), p. 26-38.

e.g. Turner, K. Tsantsanoglou, G.M. Parássoglou, "On the Derveni papyrus", Gnomon 54 (1982), p. 855 sq.

E. VANDERPOOL, "News Letter from Greece", AJA 66 (1962), p. 389-391.

F. VIAN, compte rendu de WEST (1983), in RPh 58 (1984), p. 288.

M.L. WEst, The Orphic Poems, Oxford, 1983.

-. "Hocus-Pocus in East and West: Theogony, Ritual, and the Tradition of Esoteric Commentary", in LAKs - Most (1997), p. 81-90.

N. WILSON, "Scoliasti e commentatori", SCO 33 (1983), p. 85.

-, "The relation of text and commentary in Greek books", in C. Questa, R. RaFFaELLI (éds), Il libro e il testo, Atti del Convegno Internazionale di Urbino 1982, Urbino, 1984, I, p. 105.

L.J. Zhmud', "Orficheskij papirus iz Derveni" (avec un résumé en anglais "The Orphic papyrus from Derveni"), VDI (1983), p. 118-139.

$Z P E$ (1982), "Der orphische Papyrus von Derveni", $Z P E$, 47, après p. 300, 1-12*.

* Ce travail a été possible grâce à l'aide de l'État espagnol (Proyecto de Investigación del Programa Sectorial de Promoción del Conocimiento, PB98-0763). Je veux remercier Mme María Esclavitud Rey Pereira pour la traduction française. 